\title{
Dicotomias Exponenciais e Pontos Homoclínicos
}

\author{
VANDERLEI MINORI HORITA
}

Orientador: Prof. DR. José Gaspar RuAS Filho

Dissertaçâo apresentada ao Instituto de Ciências Matemáticas de São Carlos - USP, como parte dos requisitos para obtenção do título de "Mestre em Ciências - Área: Matemática".

USP - São Carlos

Julho de 1993 
À minha família 


\begin{abstract}
Smale showed, using the famous horseshoe construction, that for diffeomorphisms with transversal homoclinic points there is a compact invariant set on which the action of some iterate of the diffeomorphism is topologically conjugate to the action of the Bernoulli shift. We will show this statement using the so-called shadowing lemma. Our basic technique is the theory of exponential dichotomies.
\end{abstract}




\section{Resumo}

Smale mostrou, usando a famosa construção do horseshoe, que para difeomorfismos com pontos homoclínicos transversais existe um conjunto invariante compacto no qual a ação de algum iterado do difeomorfismo é topologicamente conjugado à açào do shift de Bernoulli. Mostraremos este resultado utilizando o shadowing lemma. Nossa técnica básica é a teoria de dicotomias exponenciais. 


\section{Agradecimentos}

Ao Gaspar pela orientaçâo segura, pela dedicação e pela confiança; sendo ele, o responsável pelas qualidades deste trabalho (se é que possuj alguma); os erros, que são muitos, estes são totalmente devidos a mim.

À minha família pelo apoio e incentivo, embora nem sempre entendendo como alguém possa gostar de matemática.

Aos professores, que seja em cursos ou seja em conversas me ensinaram o pouco de matemática que sei.

Aos meus colegas que me suportaram e que tornaram muito agradáveis estes anos. Em particular ao Antonio e ao Marcos, pelas incontáveis "discussões", que ajudaram tornar claras idéis e conceitos.

À Beth e a Laura, pela eficiência que tratam dos nossos assuntos acadêmicos.

Aos funcionários do instituto, cuja ação nos dá condiçôes de desenvolver nosso trabalho.

Agradeço a todas as pessoas que direta ou indiretamente me ajudaram na elaboração deste trabalho. Peço desculpas àqueles que injusta e involuntariamente tenham sido omitidos.

Este trabalho teve suporte financeiro do CNPq. 


\section{Conteúdo}

Introdução

1 Dicotomias Exponenciais para Equaçōes Diferença Lineares

2 Conjuntos Hiperbólicos e o Shadowing Lemma

3 Comportamento Caótico na vizinhança de um ponto homoclínico transversal: O Teorema de Smale

4 Equivalência de Definições de Ponto Homoclínico Transversal

39

Bibliografia 


\section{Introdução}

Os difeomorfismos com pontos homoclínicos transversais foram estudados por Smale em [8] e [9]. Nestes trabalhos foi demonstrado que a dinâmica é caótica na vizinhança de tais pontos, no sentido que existe um conjunto invariante compacto no qual a ação de algum iterado do difeomorfismo é topologicamente conjugado à ação do shift de Bernoulli (hoje conhecido como Teorema de Smale). Como consequencia imediata disto temos o resultado de Birkhoff de que o difeomorfismo tem infinitos pontos periódicos. Um outro resultado que podemos obter é que difeomorfismos próximos devem também ter pontos homoclínicos transversais e, portanto, infinitos pontos periódicos. Assim, a propriedade de ter infinitos pontos periódicos não pode, em geral, ser perturbada.

Smale provou seu teorema usando a famosa construção do horseshoe (veja também Moser [3] e Niteckj [4]). Nosso trabalho é baseado em Palmer [5] e tem como objetivo principal apresentar uma demonstração, em um certo sentido "mais fácil", usando o shadowing lemma (Anosov [1], Guckenheimer, Moser e Newhouse [2], Robinson [7]) para provar o teorema. Tal prova foi esboçada em Palmer [6] e levada até o fim no caso onde o difeomorfismo é uma aplicaçâo correspondente a um sistema periódico de equações diferenciais. Nâo apenas provaremos o Teorema de Smale como também daremos uma prova do shadowing lemma. Nossa técnica básica será a teoria de dicotomias exponenciais

No capítulo 1 desenvolvemos a teoria de dicotomias exponenciais para equaçôes diferença linear. Também damos um resultado sobre a existência de soluções limitadas de sistemas quasilinear onde a parte linear tem uma dicotomia exponencial.

No capítulo 2 iniciamos o estudo de difeomorfismos. Vemos que toda órbita de difeomorfismo tem associada uma equaçâo diferença linear chamada sua equação variacional. Rigorosamente falando, um conjunto invariante é hiperbólico se a equaçâo variacional de cada órbita no conjunto tem uma dicotomia exponencial. O shadowing lemma, que é una propriedade de conjuntos hiperbólicos, passa ser 
uma consequência do resultado de sistemas quasilinear provado no capitulo 1.

No capítulo 3 os pontos homoclínicos transversais sâo definidos. A definição de transversalidade é puramente em termos da equação variacional da órbita do ponto homoclínico transversal. Usamos entâo o shadowing lemma para provar o Teorema de Smale.

Um ponto homoclínico transversal é usualmente definido como um ponto onde as variedades estável e instável de um ponto fixo hiperbólico intercectam-se transversalmente. No capítulo 4 usamos as ferramentas do capítulo 1 para provar o teorema da variedade estável e mostrar que a definição de ponto homoclínico dado no capítulo 3 é equivalente à usual. Finalmente, mostramos que próximo a qualquer ponto homoclínico transversal existem infinitos outros e também que a propriedade de ter um ponto homoclinico transversal persiste sob perturbação. 


\section{Capítulo 1}

\section{Dicotomias Exponenciais para Equações Diferença Lineares}

Neste capitulo desenvolvemos as ferramentas que servirão de base para o resto deste trabalho. Desenvolvemos a teoria de dicotomias exponenciais para equaçôes diferença linear e obtemos um resultado sobre a existência de soluções limitadas de sistemas quasilinear onde a parte linear possui uma dicotomia exponencial.

Consideraremos equações diferença lineares

$$
u_{n+1}=C_{n} u_{n}
$$

onde $u_{n} \in \mathbf{R}^{p}, n \in J$ ( $J$ é usualmente $\mathbf{Z}$, os inteiros não negativos $\mathbf{Z}^{+}$ou os inteiros não positivos $\mathbf{Z}^{-}$) e para cada $n C_{n}$ é uma matriz $p \times p$ inversivel. Para $n, m \in J$ definimos a matriz de transição

$$
\Phi(n, m)=\left\{\begin{array}{clcl}
C_{n-1} & \ldots & C_{m} & \text { se } n>m \\
I & & & \text { se } n=m \\
C_{n}^{-1} & \ldots & C_{m-1}^{-1} & \text { se } n<m
\end{array}\right.
$$

Ela tem a propriedade

$$
\Phi(n, k) \Phi(k, m)=\Phi(n, m) \quad \text { se } n, k, m \in J
$$

e a propriedade que $u_{n}=\Phi(n, m) \xi$ é a única solução da equação (1.1) safisfazendo $u_{m}=\xi$. Note também como uma consequência da propriedade que

$$
\Phi^{-1}(n, m)=\Phi(m, n) \quad \text { se } n, m \in J
$$


Definição 1.1 A equaçâo diferença linear (1.1) tem uma dicotomia exponencial em $J$ se existem constantes positivas $K, \alpha \in$ umá familia de projeçôes $P_{n}, n \in J$, tal que

(i) $P_{n+1} C_{n}=C_{n} P_{n}$ para todo $n$ ( $n<0$ se $J=\mathrm{Z}^{-}$)

(ii) $\left|\Phi(n, m) P_{m}\right| \leq K e^{-\alpha(n-m)}$ para $n \geq m$

$\left|\Phi(n, m)\left(I-P_{m}\right)\right| \leq K e^{-\alpha(m-n)}$ para $m \geq n$

Por repetidas aplicações de (i) obtemos a identidade

$$
P_{n} \Phi(n, m)=\Phi(n, m) P_{m}
$$

Isto significa que as projeções $P_{n}$ sâo invariantes com respeito à equação (1.1). Isto é, se $u_{n}$ é uma solução de (1.1) tal que $u_{m}$ está na imagem (respec. núcleo) de $P_{m}$, para algum $m$, então $u_{n}$ está na imagem (respec. núcleo) de $P_{n}$ para todo $n$. A propriedade (ii) diz, em primeiro lugar, que os $P_{n}$ 's são limitados e, em segundo, que as soluções $u_{n}$ da equação (1.1) que estâo na imagem de $P_{n}$ decaem exponencialmente enquanto as que estâo no núcleo de $P_{n}$ crescem exponencialmente.

Proposição 1.1 Notamos que se, como na Definiçâo 1.1, a equação (1.1) tem uma dicotomia exponencial em $J=[q, \infty)$, onde $q>0$, então ela tem uma dicotomia exponencial em $\mathbf{Z}^{+}$(vale também para $J=\mathbf{Z}^{-}$)

\section{Prova:}

Defina $P_{n}=\Phi(n, q) P_{q} \Phi(q, n)$ para $0 \leq n<q$. Entào a propriedade (i) na Definição 1.1 é satisfeita para $n \geq 0$, pois

$$
\begin{aligned}
P_{n+1} & =\Phi(n+1, q) P_{q} \Phi(q, n+1) \\
& =C_{n+1}^{-1} \ldots C_{q-1}^{-1} P_{q} C_{q-1} \ldots C_{n+1} \\
& =C_{n} \Phi(n, q) P_{q} \Phi(q, n) C_{n}^{-1}
\end{aligned}
$$

Sabemos que as desigualdades (1.3) e (1.4) ocorrem para $n \geq m \geq q, m \geq n \geq q$ respectivamente. Considere

$$
M_{1}=\sup \left\{\left|\Phi(n, m) P_{m}\right|: 0 \leq n, m \leq q\right\}
$$


Então se $n \geq q>m \geq 0$

$$
\begin{aligned}
\left|\Phi(n, m) P_{m}\right| & =\left|\Phi(n, q) \Phi(q, m) P_{m} P_{m}\right| \\
& =\left|\Phi(n, q) P_{q} \Phi(q, m) P_{m}\right| \\
& \leq\left|\Phi(n, q) P_{q}\right|\left|\Phi(q, m) P_{m}\right| \\
& \leq M_{1} K e^{-\alpha(n-q)} \leq M_{1} K e^{\alpha q} e^{-\alpha(n-m)}
\end{aligned}
$$

e se $q \geq n \geq m \geq 0$

$$
\left|\Phi(n, m) P_{m}\right| \leq M_{1} \leq M_{1} e^{\alpha q} e^{-\alpha(n-m)}
$$

Analogamente, defina

$$
M_{2}=\sup \left\{\left|\Phi(n, m)\left(I-P_{m}\right)\right|: 0 \leq n, \ddot{m} \leq q\right\}
$$

se $m \geq q>n \geq 0$

$$
\begin{aligned}
\left|\Phi(n, m)\left(I-P_{m}\right)\right| & =\left|\Phi(n, q) \Phi(q, m)\left(I-P_{m}\right)\left(I-P_{m}\right)\right| \\
& =\left|\Phi(n, q)\left(I-P_{q}\right) \Phi(q, m)\left(I-P_{m}\right)\right| \\
& \leq M_{2} K e^{\alpha q} e^{-\alpha(m-n)}
\end{aligned}
$$

e se $q \geq m \geq n \geq 0$

$$
\left|\Phi(n, m)\left(I-P_{m}\right)\right| \leq M_{2} \leq M_{2} K e^{\alpha q} e^{-\alpha(m-n)}
$$

Então, notando que $M_{1}, M_{2} \geq 1$, concluímos que a equação (1.1) tem uma dicotomia exponencial em $\mathrm{Z}^{+}$com constantes $\max \left\{M_{1} K e^{\alpha q}, M_{2} K e^{\alpha q}\right\}$ e $\alpha$.

Segue de (1.5) que quando $J=\mathbf{Z}, \mathbf{Z}^{+}$ou $\mathbf{Z}^{-}$entâo

$$
P_{n}=\Phi(n, 0) P_{0} \Phi(0, n)
$$

Isto mostra que o posto de $P_{n}$ não depende de $n$ e que $P_{n}$ está determinado uma vez que $P_{0}$ está. Em nossa primeira proposição determinamos todas as possíveis escolhas de $P_{0}$. Usaremos as notações $\mathcal{R}, \mathcal{N}$ para denotar a imagem e o núcleo de uma transformação linear. 
Proposição 1.2 Seja a equação diferença linear (1.1) tendo uma dicotomia exponencial em $J$ com constantes $K, \alpha$ e projeções $P_{n}$. Denote por $\Phi(n, m)$ a matriz de transiçâo para (1.1).

(i) $S e J=\mathbf{Z}^{+}$, entâo

$$
\mathcal{R}\left(P_{0}\right)=\left\{\xi \in \mathbf{R}^{p}: \sup _{n \geq 0}|\Phi(n, 0) \xi|<\infty\right\} .
$$

e se $Q_{0}$ é outra projeçâo com $\mathcal{R}\left(Q_{0}\right)=\mathcal{R}\left(P_{0}\right)$, (1.1) tem uma dicotomia exponencial em $\mathrm{Z}^{+}$com projeçôes $Q_{n}=\Phi(n, 0) Q_{0} \Phi(0, n)$,

(ii) $\mathrm{Se} J=\mathrm{Z}^{-}$, entâo

$$
\mathcal{N}\left(P_{0}\right)=\left\{\xi \in \mathbf{R}^{p}: \sup _{n \leq 0}|\Phi(n, 0) \xi|<\infty\right\}
$$

e se $Q_{0}$ é outra projeçâo com $\mathcal{N}\left(Q_{0}\right)=\mathcal{N}\left(P_{0}\right)$, (1.1) tem uma dicotomia exponencial em $\mathrm{Z}^{-}$com projeşôes $Q_{n}=\Phi(n, 0) Q_{0} \Phi(0, n)$,

(iii) $S e J=Z, \mathcal{R}\left(P_{0}\right)$ é dado por (1.7) e $\mathcal{N}\left(P_{0}\right)$ por (1.8), então $P_{0}$ e portanto os $P_{n}$ são unicamente determinados.

Prova:

(i) Se $\xi \in \mathbf{R}^{p}$ segue de (1.3), com $m=0$ que, para $n \geq 0$,

$$
\left|\Phi(n, 0) P_{0} \xi\right| \leq K e^{-a n}\left|P_{0} \xi\right|
$$

Além disso, usando (1.2), (1.4) e (1.5), .

$$
\begin{aligned}
\left|\left(I-P_{0}\right) \xi\right| & =\left|\Phi(0, n) \Phi(n, 0)\left(I-P_{0}\right)\left(I-P_{0}\right) \xi\right| \\
& =\left|\Phi(0, n)\left(I-P_{n}\right) \Phi(n, 0)\left(I-P_{0}\right) \xi\right| \\
& \leq\left|\Phi(0, n)\left(I-P_{n}\right)\right|\left|\Phi(n, 0)\left(I-P_{0}\right) \xi\right| \\
& \leq K \dot{e}^{-\alpha n}\left|\Phi(n, 0)\left(I-P_{0}\right) \xi\right|
\end{aligned}
$$

Logo, para $n \geq 0$

$$
\left|\Phi(n, 0)\left(I-P_{0}\right) \xi\right| \geq K^{-1} e^{\alpha n}\left|\left(I-P_{0}\right) \xi\right|
$$

"Portanto, $\Phi(n, 0) \xi=\Phi(n, 0) P_{0} \xi+\Phi(n, 0)\left(I-P_{0}\right) \xi$ é limitado se, e somente se, $\left(I-P_{0}\right) \xi=0$, isto é, se, e somente se, $\xi \in \mathcal{R}\left(P_{0}\right)$. Isto estabelece (1.7).

Agora, seja $Q_{0}$ qualquer projeção com $\mathcal{R}\left(Q_{0}\right)=\mathcal{R}\left(P_{0}\right)$ e defina

$$
Q_{n}=\Phi(n, 0) Q_{0} \Phi(0, n)
$$


Então para $n \geq m \geq 0$

$$
\begin{aligned}
\left|\Phi(n, m)\left(Q_{m}-P_{m}\right)\right| & =\left|\Phi(n, m) \Phi(m, 0)\left(Q_{0}-P_{0}\right) \Phi(0, m)\right| \\
& =\left|\Phi(n, 0)\left(Q_{0}-P_{0}\right) \Phi(0, m)\right| \\
& =\left|\Phi(n, 0) P_{0}\left(Q_{0}-P_{0}\right)\left(I-P_{0}\right) \Phi(0, m)\right| \\
& =\left|\Phi(n, 0) P_{0}\left(Q_{0}-P_{0}\right) \Phi(0, m)\left(I-P_{m}\right)\right| \\
& \leq\left|\Phi(n, 0) P_{0}\right|\left|Q_{0}-P_{0}\right|\left|\Phi(0, m)\left(I-P_{m}\right)\right| \\
& \leq K^{2} e^{-\alpha(n+m)}\left|Q_{0}-P_{0}\right|
\end{aligned}
$$

onde a terceira igualdade decorre do fato que $\mathcal{R}\left(Q_{0}\right)=\mathcal{R}\left(P_{0}\right)$ assim, para $n \geq m \geq 0$

$$
\begin{aligned}
\left|\Phi(n, m) Q_{m}\right| & \leq\left|\Phi(n, m) P_{m}\right|+\left|\Phi(n, m)\left(Q_{m}-P_{m}\right)\right| \\
& \leq K|1+K| Q_{0}-P_{0} \| e^{-\alpha(n-m)}
\end{aligned}
$$

Analogamente, para $m \geq n \geq 0$

$$
\left|\Phi(n, m)\left(I-Q_{m}\right)\right| \leq K\left[1+K\left|Q_{0}-P_{0}\right|\right] e^{-\alpha(m-n)}
$$

Finalmente, observamos que a invariância de $Q_{n}$ com respeito a equação (1.1) segue de sua definição e portanto, completa a prova de (i).

(ii) Se $\xi \in \mathbf{R}^{p}$ segue de (1.4), com $m=0$, que para $n \leq 0$, temos

$$
\left|\Phi(n, 0)\left(I-P_{0}\right) \xi\right| \leq K e^{\alpha n}\left|\left(I-P_{0}\right) \xi\right|
$$

Além disso, usando (1.2), (1.3) e (1.5) segue

$$
\begin{aligned}
\left|P_{0} \xi\right| & =\left|\Phi(0, n) \Phi(n, 0) P_{0} P_{0} \xi\right| \\
& =\left|\Phi(0, n) P_{n} \Phi(n, 0) P_{0} \xi\right| \\
& \leq\left|\Phi(0, n) P_{n}\right|\left|\Phi(n, 0) P_{0} \xi\right| \\
& \leq K e^{\alpha n}\left|\Phi(n, 0) P_{0} \xi\right|
\end{aligned}
$$

Assim, para $n \leq 0$,

$$
\left|\Phi(n, 0) P_{0} \xi\right| \geq K e^{-\alpha n}\left|P_{0} \xi\right|
$$

Portanto $\Phi(n, 0) \xi=\Phi(n, 0) P_{0} \xi+\Phi(n, 0)\left(I-P_{0}\right) \xi$ é limitado se, e somente se, $P_{0} \xi=0$, isto é, se, e somente se, $\xi \in \mathcal{N}\left(P_{0}\right)$. Isto estabelece (1.8).

Agora, seja $Q_{0}$ qualquer projeçâo $\operatorname{com} \mathcal{N}\left(P_{0}\right)=\mathcal{N}\left(Q_{0}\right)$ e defina

$$
Q_{n}=\Phi(n, 0) Q_{0} \Phi(0, n) .
$$


Então, para $m \leq n \leq 0$

$$
\begin{aligned}
\left|\Phi(n, m)\left(Q_{m}-P_{m}\right)\right| & =\left|\Phi(n, m) \Phi(m, 0)\left(Q_{0}-P_{0}\right) \Phi(0, m)\right| \\
& =\left|\Phi(n, 0)\left(Q_{0}-P_{0}\right) \Phi(0, m)\right| \\
& =\left|\Phi(n, 0)\left(I-P_{0}\right)\left(Q_{0}-P_{0}\right) P_{0} \Phi(0, m)\right| \\
& =\left|\Phi(n, 0)\left(I-P_{0}\right)\left(Q_{0}-P_{0}\right) \Phi(0, m) P_{m}\right| \\
& \leq\left|\Phi(n, 0)\left(I-P_{0}\right)\right|\left|\left(Q_{0}-P_{0}\right)\right|\left|\Phi(0, m) P_{m}\right| \\
& \leq K e^{\alpha n}\left|Q_{0}-P_{0}\right| K \epsilon^{\alpha m} \\
& =K^{2} e^{\alpha(n+m)}\left|Q_{0}-P_{0}\right|
\end{aligned}
$$

assim, para $m \leq n \leq 0$,

$$
\begin{aligned}
\left|\Phi(n, m) Q_{m}\right| & \leq\left|\Phi(n, m) P_{m}\right|+\left|\Phi(n, m)\left(Q_{m}-P_{m}\right)\right| \\
& \leq K e^{-\alpha(n-m)}+K^{2} e^{\alpha(n+m)}\left|Q_{0}-P_{0}\right| \\
& \leq K\left[1+K \mid Q_{0}-P_{0} \| e^{-\alpha(n-m)}\right.
\end{aligned}
$$

Analogamente, para $n \leq m \leq 0$,

$$
\left|\Phi(n, m)\left(I-Q_{m}\right)\right| \leq K\left[1+K\left|Q_{0}-P_{0}\right|\right] \epsilon^{-\alpha(m-n)}
$$

A invariância de $Q_{n}$ com relação a equaçâo (1.1) segue de sua definição e assim a prova de (ii).

(iii) De (i) e (ii), temos que $\mathcal{R}\left(P_{0}\right)$ e $\mathcal{N}\left(P_{0}\right)$ ficam unicamente determinados, ou seja, $P_{0}$ e consequentemente $P_{n}$, ficam bem determinados.

Observação 1.1 Segue da Proposiçâo 1.2 que quando $J=\mathrm{Z}^{+}$(respectivamente $J=\mathbf{Z}^{-}$), a única solução limitada $u_{n}$ da equação (1.1) em $J$ com $P_{0} u_{0}=0$ (respectivamente $\left.\left(I-P_{0}\right) u_{0}=0\right)$ é a trivial; e, quando $J=\mathbf{Z}$, a única solução limitada de (1.1) é a trivial.

De fato, mostraremos para o caso $J=\mathrm{Z}^{+}$, o outro segue por argumentos análogos. Seja $u_{n}$ uma solução limitada de 1.1 , isto é,

$$
\sup _{n \geq 0}\left|u_{n}\right|<\infty
$$

Mas, $u_{n}=\Phi(n, 0) u_{0}$, ou seja

$$
\sup _{n \geq 0}\left|\Phi(n, 0) u_{0}\right|<\infty
$$

Assim, $u_{0} \in \mathcal{R}\left(P_{0}\right)$, logo $0=P_{0} u_{0}=u_{0}$. Portanto, $u_{n}$ deve ser trivial. 
Exemplo 1 Quando $C_{n}$ independe de $n$ a equaşão 1.1 tem uma dicotomia exponencial em $J$ se, e somente se, os autovalores de $C$ não estäo no círculo unitário.

De fato, se $C$ satisfaz a última condição, denotamos por $P$ a projeção com $\mathcal{R}(P)=\{$ soma dos autoespaços generalizados de $C$ correspondentes aos autovalores dentro do círculo unitário\}

e com

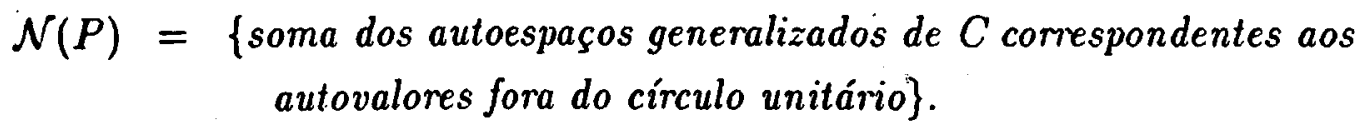

Então $C$ comuta com $P$ e existem constantes positivas $K$ e $\alpha$ tal que

$$
\begin{gathered}
\left|C^{n} P\right| \leq K e^{-\alpha n} \quad(n \geq 0), \\
\left|C^{n}(I-P)\right| \leq K e^{\alpha n} \quad(n \leq 0) .
\end{gathered}
$$

Isto significa que a equação

$$
u_{n+1}=C u_{n}
$$

que tem matriz de transição $C^{n-m}$ tem uma dicotomia exponencial em $\mathbf{Z}$ com projeções constantes $P$.

Reciprocamente, suponha que a última equação tem uma dicotomia exponencial em $\mathbf{Z}$ com constantes $K, \alpha$ e projeçôes $P_{n}$. Logo

$$
\begin{gathered}
\left|C^{n-m} P_{m}\right| \leq K e^{-\alpha(n-m)} \quad(n \geq m) \\
\left|C^{n-m}\left(I-P_{m}\right)\right| \leq K e^{-\alpha(m-n)} \quad(m \geq n) .
\end{gathered}
$$

Se definirmos $Q_{n}=P_{n+1}$, vemos que

$$
\begin{gathered}
\left|C^{n-m} Q_{m}\right|=\left|C^{n+1-(m+1)} P_{m+1}\right| \leq K \epsilon^{-\alpha(n-m)} \quad(n \geq m), \\
\left|C^{n-m}\left(I-Q_{m}\right)\right|=\left|C^{n+1-(m+1)}\left(I-P_{m+1}\right)\right| \leq K e^{-\alpha(m-n)} \quad(m \geq n) .
\end{gathered}
$$

Como $P_{n}$ é invariante, temos para todo $n$,

$$
Q_{n+1} C=P_{n+2} C=C P_{n+1}=C Q_{n}
$$

Assim, também temos uma dicotomia exponencial com projeçôes $Q_{n}$. Segue pela Proposição 1.2 (iii) que $Q_{n}=P_{n}$ para todo $n$. Portanto $P_{n}$ é, na verdade, uma 
constante $P$ (digamos). Então segue da invariância de $P_{n}$ que $P$ comuta com $C$ e, além disso, das desigualdades acima, que

$$
\begin{gathered}
\left|C^{n} P\right| \leq K e^{-a n} \quad(n \geq 0) \\
\left|C^{n}(I-P)\right| \leq K e^{\alpha n} \quad(n \leq 0)
\end{gathered}
$$

Isto significa que $\mathbf{R}^{p}=\mathcal{R}(P) \oplus \mathcal{N}(P)$ onde $\mathcal{R}(P)$ e $\mathcal{N}(P)$ são ambos invariantes por $C$ e os autovalores de $C$ restritos a $\mathcal{R}(P)$ estão dentro do círculo unitário e aqueles de $C$ restritos $\mathcal{N}(P)$ estão fora do círculo unitário. Portanto, todos os autovalores de $C$ nâo pertencem ao círculo unitário.

É claro que quando (1.1) tem uma dicotomia exponencial em $\mathbf{Z}$ então tem uma em $\mathbf{Z}^{+}$e uma em $\mathbf{Z}^{-}$. Em nossa próxima proposição examinamos a extensão para o qual a recíproca deste comportamento é verdadeira.

Proposição 1.3 Seja $C_{n}$ uma funçâo matriz $p \times p$ inversivel definida para todo n. Entâo a equação diferença linear (1.1) tem um dicotomia exponencial em $\mathbf{Z}$ se, e somente se, ela tem uma em $\mathbf{Z}^{+}$e uma $\mathrm{em} \mathbf{Z}^{-}$com projeçóes do mesmo posto, e a única soluçâo limitada em $\mathbf{Z}$ é a trivial.

\section{Prova:}

$(\Rightarrow)$ Segue da definição de dicotomia exponencial e da Observação 1.1 .

$(\Leftrightarrow)$ Suponha que $(1.1)$ tenha uma dicotomia exponencial em $\mathbf{Z}^{+}$e em $\mathbf{Z}^{-}$com projeções $P_{n}$ e $Q_{n}$, respectivamente, ambas com mesmo posto, e que ela tenha como única solução limitada em $\mathbf{Z}$ a soluçâo trivial. Pela. Proposiçâo 1.2 podemos escolher $P_{0}$ como alguma projeção com imagem

$$
V^{+}=\left\{\xi \in \mathbf{R}^{p}: \sup _{n \geq 0}|\Phi(n, 0) \xi|<\infty\right\}
$$

e $Q_{0}$ como alguma projeção com núcleo

$$
V^{-}=\left\{\xi \in \mathbf{R}^{p}: \sup _{n \leq 0}|\Phi(n, 0) \xi|<\infty\right\}
$$

[Aqui $\Phi(n, m)$ é a matriz de transição de (1.1)]. Como a única solução limitada de (1.1) em $\mathbf{Z}$ é a trivial, $V^{+} \cap V^{-}=\{0\}$ e como $P_{0}$ e $Q_{0}$ tem o mesmo posto, $\operatorname{dim} V^{+}+\operatorname{dim} V^{-}=p$. Logo $\mathbf{R}^{p}=V^{+} \oplus V^{-}$e portanto, podemos escolher $P_{0}$ e $Q_{0}$ como a mesma projeção ; com imagem $V^{+}$e núcleo $V^{-}$. Entâo uma 
desigualdade como (1.3) ocorre para $n \geq m \geq 0,0 \geq n \geq m$ e outra como (1.4) para $m \geq n \geq 0,0 \geq m \geq n$. Se $n \geq 0 \geq m$, temos

$$
\begin{aligned}
\left|\Phi(n, m) P_{m}\right| & =\left|\Phi(n, m) \Phi(m, 0) P_{0} \Phi(0, m)\right| \\
& =\left|\Phi(n, 0) P_{0} P_{0} \Phi(0, m)\right| \\
& =\left|\Phi(n, 0) P_{0} \Phi(0, m) P_{m}\right| \\
& \leq\left|\Phi(n, 0) P_{0}\right|\left|\Phi(0, m) P_{m}\right| \\
& \leq K e^{-\alpha n} K e^{\alpha m}=K^{2} e^{-\alpha(n-m)}
\end{aligned}
$$

e se $n \leq 0 \leq m$, temos

$$
\begin{aligned}
\left|\Phi(n, m)\left(I-P_{m}\right)\right| & =\left|\Phi(n, m) \Phi(m, 0)\left(I-P_{0}\right) \Phi(0, m)\right| \\
& =\left|\Phi(n, 0)\left(I-P_{0}\right)\left(I-P_{0}\right) \Phi(0, m)\right| \\
& =\left|\Phi(n, 0)\left(I-P_{0}\right) \Phi(0, m)\left(I-P_{m}\right)\right| \\
& \leq\left|\Phi(n, 0)\left(I-P_{0}\right)\right|\left|\Phi(0, m)\left(I-P_{m}\right)\right| \\
& \leq K e^{-\alpha m} K e^{a n}=K^{2} e^{-\alpha(m-n)}
\end{aligned}
$$

Portanto, (1.1) tem uma dicotomia exponencial em Z.

Nosso próximo objetivo é provar a existência de um solução limitada de uma equação difereça quasilinear. Primeiro consideraremos uma equação não homogênea.

Lema 1.1 Considere a equação diferença linear (1.1) com um dicotomia exponencial em $J$ com constantes $K, \alpha$ e projeçôes $P_{n}$. Seja $h_{n}, n \in J$ uma sequência limitada de vetores.

(i) Se $J=\mathbf{Z}$ então a equação diferença nâo homogênea

$$
u_{n+1}=C_{n} u_{n}+h_{n}
$$

tem uma única solução $u_{n}$ limitada em $\mathbf{Z}$. Além disso para todo $n$,

$$
\left|u_{n}\right| \leq K^{\prime}\left(1+e^{-\alpha}\right)\left(1-e^{-\alpha}\right)^{-1} \sup _{n \in J}\left|h_{n}\right|
$$

(ii) Se $J=\mathbf{Z}^{+}$ou $\mathbf{Z}^{-}$e $\xi \in \mathbf{R}^{p}$, então (1.9) tem uma única soluçâo $u_{n}$ limitada em $J$ e satisfaz

$$
P_{0} u_{0}=P_{0} \xi \quad\left(J=\mathbf{Z}^{+}\right)
$$




$$
\left(I-P_{0}\right) u_{0}=\left(I-P_{0}\right) \xi \quad\left(J=Z^{-}\right)
$$

Além disso para n em $\mathrm{J}$,

$$
\left|u_{n}\right| \leq K^{\prime}|\xi|+K^{\prime}\left(1+\epsilon^{-\alpha}\right)\left(1-e^{-\alpha}\right)^{-1} \sup _{n \in J}\left|h_{n}\right|
$$

\section{Prova:}

(i) Denote por $\Phi(n, m)$ a matriz de transição para a equação (1.1). Então definimos

$$
u_{n}=\sum_{m \in J} G(n, m+1) h_{m} \quad \text { para } n \in J
$$

onde $G(n, m)$ é a função de Green definida por

$$
G(n, m)=\left\{\begin{array}{cc}
\Phi(n, m) P_{m} & \text { se } m \leq n \\
-\Phi(n, m)\left(I-P_{m}\right) & \text { se } n<m
\end{array}(n, m \in J)\right.
$$

Pelas desigualdades (1.3) e (1.4),

$$
|G(n, m)| \leq K e^{-\alpha|n-m|}, \quad \text { para } n, m \in J
$$

logo,

$$
\begin{aligned}
\sum_{m \in J}\left|G(n, m+1) h_{m}\right| & \leq K \sum_{m \in J} e^{-\alpha|n-m-1|} \sup _{n \in J}\left|h_{n}\right| \\
& \leq K\left(1+e^{-\alpha}\right)\left(1-e^{-\alpha}\right)^{-1} \sup _{n \in J}\left|h_{n}\right|
\end{aligned}
$$

assim

$$
\begin{aligned}
\left|u_{n}\right| & =\left|\sum_{m \in J} G(n, m+1) h_{m}\right| \\
& \leq \sum_{m \in J}\left|G(n, m+1) h_{m}\right| \\
& \leq K\left(1+\epsilon^{-\alpha}\right)\left(1-e^{-\alpha}\right)^{-1} \sup _{n \in J}\left|h_{n}\right|
\end{aligned}
$$

Portanto $u_{n}$ está bem definida e satisfaz (1.10).

Agora, note que para cada $m$ em $J$ fixado, $X_{n}=G(n, m)$ resolve a equação diferença

$$
X_{n+1}=C_{n} X_{n}+H_{n}^{(m)}
$$

onde

$$
H_{n}^{(m)}= \begin{cases}I & \text { se } n=m-1 \\ 0 & \text { se } n \neq m-1\end{cases}
$$

pois, 
- Se $m \leq n$,

$$
\begin{aligned}
C_{n} X_{n}+H_{n}^{(m)} & =C_{n} G(n, m)=C_{n} \Phi(n, m) P_{m} \\
& =\Phi(n+1, m) P_{m}=X_{n+1}
\end{aligned}
$$

- Se $n=m-1$,

$$
\begin{aligned}
C_{n} X_{n}+H_{n}^{(m)} & =C_{m-1} G(m-1, m)+I \\
& =-C_{m-1} \Phi(m-1, m)\left(I-P_{m}\right)+I \\
& =-C_{m-1} C_{m-1}^{-1}\left(I-P_{m}\right)+I \\
& =P_{m}=\Phi(m, m) P_{m}=G(m, m) \\
& =G(n+1, m)=X_{n+1}
\end{aligned}
$$

- Se $n<m-1$,

$$
\begin{aligned}
C_{n} X_{n}+H_{n}^{(m)} & =C_{n} G(n, m)=-C_{n} \Phi(n, m)\left(I-P_{m}\right) \\
& =-\Phi(n+1, m) P_{m}=G(n+1, m)=X_{n+1}
\end{aligned}
$$

Assim,

$$
\begin{aligned}
u_{n+1}= & \sum_{m \in J} G(n+1, m+1) h_{m} \\
= & \sum_{m \in J, m \leq n-1} C_{n} G(n, m+1) h_{m}+\left[C_{n} G(n, n+1)+I\right] h_{n} \\
& +\sum_{m \in J, m \geq n+1} C_{n} G(n, m+1) h_{m} \\
= & C_{n} \sum_{m \in J} G(n, m+1) h_{m}+h_{n} \\
= & C_{n} u_{n}+h_{n}
\end{aligned}
$$

Portanto, $u_{n}$ é uma solução de (1.9). Finalmente, note que a unicidade segue da Observação 1.1, pois se $v_{n}$ é outra soluçâo , então $w_{n}=u_{n}-v_{n}$ é solução da equação diferença homogênea, logo que $u_{n}=v_{n}$.

(ii) No lugar de (1.12), cosidere

$$
u_{n}=\Phi(n, 0) P_{0} \xi+\sum_{m=0}^{\infty} G(n, m+1) h_{m}
$$

quando $J=\mathrm{Z}^{+} \mathrm{e}$

$$
u_{n}=\Phi(n, 0)\left(I-P_{0}\right) \xi+\sum_{m=-\infty}^{-1} G(n, m+1) h_{m}
$$


quando $J=\mathbf{Z}^{-}$.

A prova que $u_{n}$ é uma solução de (1.9) e satisfaz a estimativa (1.11) é essencialmente a mesma da parte (i).

Quando $J=\mathbf{Z}^{+}$,

$$
\begin{aligned}
u_{0} & =P_{0} \xi+\sum_{m=0}^{\infty} G(0, m+1) h_{m} \\
& =P_{0} \xi-\sum_{m=0}^{\infty} \Phi(0, m+1)\left(I-P_{m+1}\right) h_{m} \\
& =P_{0} \xi-\sum_{m=0}^{\infty}\left(I-P_{0}\right) \Phi(0, m+1) h_{m}
\end{aligned}
$$

logo,

$$
\begin{aligned}
P_{0} u_{0} & =P_{0}\left(P_{0} \xi-\sum_{m=0}^{\infty}\left(I-P_{0}\right) \Phi(0, m+1) h_{m}\right) \\
& \left.=P_{0} \xi-P_{0} \sum_{m=0}^{\infty}\left(I-P_{0}\right) \Phi(0, m+1) h_{m}\right) \\
& \left.=P_{0} \xi-\sum_{m=0}^{\infty} P_{0}\left(I-P_{0}\right) \Phi(0, m+1) h_{m}\right) \\
& =P_{0} \xi .
\end{aligned}
$$

Analogamente, quando $J=\mathbf{Z}^{-}$, podemos mostrar que $\left(I-P_{0}\right) u_{0}=\left(I-P_{0}\right) \xi$. Finalmente, a unicidade segue da Observação 1.1 .

Proposição 1.4 Suponhamos que equação diferença linear (1.1) tenha uma dicotomia exponencial em $J$ com constantes $K$, $\alpha$ e projeçôєs $P_{n}$ e, para cada $n \mathrm{em}$ $J$, seja $g_{n}(x)$ uma função com valores em $\mathbf{R}^{p}$ próximo de 0 tal que

$$
g_{n}(0)=0, \quad\left|g_{n}\left(x_{1}\right)-g_{n}\left(x_{2}\right)\right| \leq \gamma\left|x_{1}-x_{2}\right|
$$

onde $\left|x_{1}\right| \leq \Delta,\left|x_{2}\right| \leq \Delta$. Suponhamos também que

$$
2 \Lambda^{\prime}\left(1+e^{-\alpha}\right)\left(1-e^{-\alpha}\right)^{-1} \gamma \leq 1
$$

(i) Seja $J=\mathbf{Z}$ e $h_{n}, n \in \mathbf{Z}$, uma sequência limitada de vetores satisfazendo

$$
2 K^{\prime}\left(1+e^{\alpha}\right)\left(1-e^{-\alpha}\right)^{-1}\left|h_{n}\right| \leq \Delta .
$$


para todo n. Entào a equação diferença nâo linear

$$
u_{n+1}=C_{n} u_{n}+h_{n}+g_{n}\left(u_{n}\right)
$$

tem uma única soluçâo $u_{n}$ tal que $\left|u_{n}\right| \leq \Delta$ para todo $n$, e, além disso, para todo $n$,

$$
\left|u_{n}\right| \leq 2 K\left(1+e^{-\alpha}\right)\left(1-e^{-a}\right)^{-1} \sup _{n \in J}\left|h_{n}\right|
$$

(ii) Seja $J=\mathbf{Z}^{+}$ou $\mathbf{Z}^{-}$e considere $\xi \in \mathbf{R}^{p}$ satisfazendo $|\xi| \leq \Delta / 4 K$ e $h_{n}$, $n \in J$, uma sequência limitada de vetores satisfazendo

$$
4 K\left(1+e^{-\alpha}\right)\left(1-e^{-\alpha}\right)\left|h_{n}\right| \leq \Delta
$$

para todo $n$. Entâo a equaçâo (1.14) tem uma única solução $u_{n}$ tal que $\left|u_{n}\right| \leq \Delta$ para todo $n e$

$$
\begin{aligned}
P_{0} u_{n} & =P_{0} \xi \quad\left(J=\mathbf{Z}^{+}\right) \\
\left(I-P_{0}\right) u_{n} & =\left(I-P_{0}\right) \xi \quad\left(J=\mathbf{Z}^{-}\right)
\end{aligned}
$$

Além disso, para todo n,

$$
\left|u_{n}\right| \leq 2 K^{\prime}|\xi|+2 K^{\prime}\left(1+e^{-\alpha}\right)\left(1-\epsilon^{-\alpha}\right)^{-1} \sup _{n \in J}\left|h_{n}\right|
$$

\section{Prova:}

(i) Denote por $l^{\infty}(J)$ o espaço de Banach das sequências limitadas $u=\left\{u_{n}\right\}_{n \in J}$ com valores em $\mathbf{R}^{p}$ com a norma

$$
\|u\|=\sup _{n \in J}\left|u_{n}\right|
$$

Então

$$
\mathcal{D}=\left\{u \in l^{\infty}(J):\|u\| \leq \Delta\right\}
$$

é um subconjunto fechado de $l^{\infty}(J)$ e portanto um espaço métrico completo.

Definiremos uma aplicação de $\mathcal{D}$ em $\mathcal{D}$. Para $u \in \mathcal{D}$, do Lema 1.1 a equação não homogênea

$$
\hat{u}_{n+1}=C_{n} \hat{u}_{n}+\left[h_{n}+g_{n}\left(u_{n}\right)\right]
$$

tem uma única soluçâo limitada $\hat{u}_{n}$. Além disso, por (1.10)

$$
\begin{aligned}
\|\hat{u}\| & \leq K\left(1+e^{-\alpha}\right)\left(1-\epsilon^{-\alpha}\right)^{-1} \sup _{n \in J}\left|h_{n}+g_{n}\left(u_{n}\right)\right| \\
& \leq K\left(1+e^{-a}\right)\left(1-\epsilon^{-\alpha}\right)^{-1}(\|h\|+\gamma\|u\|) \\
& \leq \frac{\Delta}{2}+\frac{\|u\|}{2} \leq \Delta
\end{aligned}
$$


Logo, a aplicaçào $u \rightarrow \hat{u}$ certamente aplica $\mathcal{D}$ em $\mathcal{D}$.

Além disso, a aplicação é uma contração, pois se $u_{1}, u_{2} \in \mathcal{D}$ e $\hat{u}_{1}, \hat{u}_{2}$ são suas imagens, entào $w_{n}=\hat{u}_{1_{n}}-\hat{u}_{2_{n}}$ é uma soluçâo limitada da equação

$$
w_{n+1}=C_{n} w_{n}+\left[g_{n}\left(u_{1_{n}}\right)-g_{n}\left(u_{2_{n}}\right)\right]
$$

Assim, novamente por (1.10),

$$
\begin{aligned}
\left\|\hat{u}_{1}-\hat{u}_{2}\right\|=\|w\| & \leq K\left(1+e^{-\alpha}\right)\left(1-\epsilon^{-\alpha}\right)^{-1} \sup _{n \in J}\left|g_{n}\left(u_{1_{n}}\right)-g_{n}\left(u_{2_{n}}\right)\right| \\
& \leq K\left(1+e^{-\alpha}\right)\left(1-e^{-\alpha}\right)^{-1} \gamma\left\|u_{1}-u_{2}\right\| \\
& \leq \frac{1}{2}\left\|u_{1}-u_{2}\right\|
\end{aligned}
$$

Denote por $u$ o único ponto fixo da aplicação $u \rightarrow \hat{u}$ em $\mathcal{D}$. Então claramente $u_{n}$ é uma solução de (1.14) satisfazendo $\left|u_{n}\right| \leq \Delta$. Além disso, ela é única, pois qualquer tal solução deve estar em $\mathcal{D}$ e ser um ponto fixo da aplicação. Finalmente, colocando $u=\hat{u} \mathrm{em}(1.16)$, obtemos

$$
\|u\| \leq K\left(1+e^{-\alpha}\right)\left(1-e^{-\alpha}\right)^{-1}\|h\|+\frac{1}{2}\|u\| .
$$

logo,

$$
\|u\| \leq 2 K^{\prime}\left(1+e^{-\alpha}\right)\left(1-e^{-\alpha}\right)^{-1}\|h\|
$$

completando a prova de (i).

(ii) A prova de (ii) é muito parecida exceto que usamos o Lema 1.1 (ii) e (1.11) no lugar do Lema 1.1 (i) e (1.10). $\hat{u}_{n}$ está definido como a única soluçâo limitada de (1.15) tal que

$$
\begin{aligned}
P_{0} u_{n} & =P_{0} \xi \quad\left(J=\mathbf{Z}^{+}\right) \\
\left(I-P_{0}\right) u_{n} & =\left(I-P_{0}\right) \xi \quad\left(J=\mathbf{Z}^{-}\right)
\end{aligned}
$$

Observação 1.2 Se $g_{n}(x)=D_{n} x$, onde $D_{n}$ é uma matriz com $\left|D_{n}\right| \leq \gamma$, obtemos um resultado similar. $\gamma$ satisfaz a mesma restrição, mas precisaremos apenas assumir que $h_{n}$ é limitada. Quando $J=\mathbf{Z}$ encontramos uma única soluçâo limitada $u_{n}$ e quando $J=\mathbf{Z}^{+}$ou $\mathbf{Z}^{-}$uma única solução limitada tal que $P_{0} u_{0}=P_{0} \xi\left(J=\mathbf{Z}^{+}\right)$e assim por diante, onde $\xi$ é qualquer vetor em $\mathbf{R}^{p}$. 
Proposição 1.5 Seja $C_{n}$ uma funçâo matriz $p \times p$ inversivel definida para $n \in J$ tal gue para todo $n$

$$
\left|C_{n}^{-1}\right| \leq M
$$

e tal que a equaçào diferença linear (1.1) tenha uma dicotomia exponencial com constantes $K, \alpha$ e projeçóes $P_{n}$. Suponha $0<\delta<\alpha$ e que $D_{n}$ é uma função matriz $p \times p$ definida para $n \in J$ e satisfazendo

$$
\begin{gathered}
\left|D_{n}\right|<M^{-1}, \\
2 K\left(1+e^{-\alpha}\right)\left(1-e^{-\alpha}\right)\left|D_{n}\right| \leq 1, \\
2 K e^{\alpha}\left(e^{-\delta}+1\right)\left(e^{\delta}-1\right)^{-1}\left|D_{n}\right| \leq 1 .
\end{gathered}
$$

Entâo $C_{n}+D_{n}$ é inversivel para todo ne a equaçâo diferença linear pertubada

$$
u_{n+1}=\left(C_{n}+D_{n}\right) u_{n}
$$

tem uma dicotomia exponencial em $J$ com constantes $2 K\left(1+e^{\delta}\right)\left(1-e^{-\delta}\right)^{-1}, \alpha-\delta$ e projeçôes de posto igual à de (1.1)

\section{Prova:}

Para todo $n$ temos

$$
\left|\left(C_{n}+D_{n}\right)-C_{n}\right|=\left|D_{n}\right|<M^{-1} \leq\left|C_{n}^{-1}\right|^{-1}
$$

Logo $C_{n}+D_{n}$ é inversivel para todo $n$. [Note que as condiçôes $\left|C_{n}^{-1}\right| \leq M$, $\left|D_{n}\right|<M^{-1}$ são usadas apenas para assegurar a inversibilidade de $C_{n}+D_{n}$ ]. Agora denote por $\Phi(n, m)$ a matriz de transiçâo para a equação (1.1). Para cada $m$ em $J$ definimos a sequência

$$
H_{n}^{(m)}=\left\{\begin{array}{ll}
I & \text { se } n=m-1 \\
0 & \text { se } n \neq m-1
\end{array} \quad(n \in J)\right.
$$

e seja $X_{n}=G(n, m)$ a única solução limitada da equaçâo

$$
X_{n+1}=\left(C_{n}+D_{n}\right) X_{n}+H_{n}^{(m)}
$$

que satisfaz

$$
\begin{gathered}
P_{0} X_{0}=0 \text { quando } J=\mathrm{Z}^{+} \text {e } m \geq 1 \\
P_{0} X_{0}=P_{0} \text { quando } J=\mathbf{Z}^{+} \text {e } m=0 \\
\left(I-P_{0}\right) X_{0}=0 \text { quando } J=\mathbf{Z}^{-}
\end{gathered}
$$


Isto é possivel pela Proposição 1.4 e Observaçâo 1.2. Aém disso

$$
|G(n, m)| \leq 2 K\left(1+e^{-\alpha}\right)\left(1-\epsilon^{-\alpha}\right)^{-1}
$$

para $n, m$ em $J$. [Note que quando $J=\mathbf{Z}^{+}$e $m=0, H_{n}^{(m)}=0$ para todo $n$ e que $\left.|G(n, 0)| \leq 2 K^{\prime}\right]$. O resto da prova consiste em mostrar que $G(n, m)$ é uma 'função de Green' para a equaçâo (1.17) (cf. a prova do Lema 1.1).

Para um $m$ fixado em $J$ considere a equação modificada

$$
X_{n+1}=e^{\ell_{n}^{(m)}(\alpha-\delta)}\left(C_{n}+D_{n}\right) X_{n}+H_{n}^{(m)},
$$

onde

$$
\epsilon_{n}^{(m)}=\left\{\begin{array}{cc}
1 & \text { se } n \geq m \\
-1 & \text { se } n<m
\end{array}\right.
$$

Esta equação pode ser reescrita como

$$
X_{n+1}=e^{\epsilon_{n}^{(m)}(\alpha-\delta)} C_{n} X_{n}+e^{\epsilon_{n}^{(m)}(\alpha-\delta)} D_{n} X_{n}+H_{n}^{(m)}
$$

Note que para $m$ fixo, a matriz de transição da equaçâo $u_{n+1}=\tilde{C}_{n} u_{n}$ onde $\tilde{C}_{n}=e^{e_{n}^{(m)}(\alpha-\delta)} C_{n} \dot{\mathbf{e}}:$

$$
\hat{\Phi}(n, m)= \begin{cases}e^{\epsilon_{n}^{(m)}(\alpha-\delta)(n-m)} \Phi(n, m) & \text { se } n \geq m \\ e^{\epsilon_{n}^{(m)}(\alpha-\delta)(m-n)} \Phi(n, m) & \text { se } n<m\end{cases}
$$

assim,

$$
\begin{aligned}
& \text { (i) } P_{n+1} \tilde{C}_{n}=P_{n+1} e^{\epsilon_{n}^{(m)}(a-\delta)} C_{n}=e^{\epsilon_{n}^{(m)}(\alpha-\delta)} P_{n+1} C_{n} \\
& =e^{\epsilon_{n}^{(m)}(\alpha-\delta)} C_{n} P_{n}=\hat{C}_{n} P_{n} \\
& \text { (ii) }\left|\tilde{\Phi}(n, m) P_{m}\right|=\left|e^{\epsilon_{n}^{(m)}(a-\delta)(n-m)} \Phi(n, m) P_{m}\right| \\
& \leq e^{c_{n}^{(m)}(\alpha-\delta)(n-m)} K e^{-\alpha(n-m)} \\
& =K e^{-\delta(n-m)} \text { para } n \geq m \\
& \left|\tilde{\Phi}(n, m)\left(I-P_{m}\right)\right|=\left|e^{\epsilon_{n}^{(m)}(a-\delta)(m-n)} \Phi(n, m)\left(I-P_{m}\right)\right| \\
& \leq e^{\ell_{n}^{(m)}(a-\delta(m-n)} K e^{-\alpha(m-n)} \\
& =K e^{-\delta(m-n)} \text { para } m>n
\end{aligned}
$$

Portanto, a equação

$$
u_{n+1}=\epsilon^{\epsilon_{n}^{(m)}(\alpha-\delta)} C_{n} u_{n}
$$

tem uma dicotomia exponencial em $J$ com constantes $K$, $\delta$ e projeçôes $P_{n}$. Logo, novamente pela Proposição 1.4 e Observação 1.2, a equação (1.19) tem uma única 
solução limitada $X_{n}=G_{\delta}(n, m)$ satisfazendo $P_{0} X_{0}=0$ quando $J=Z^{+}$e $m \geq 1$ e assim por diante. Além disso, temos a estimativa

$$
\left|G_{\delta}(n, m)\right| \leq 2 K\left(1+e^{-\delta}\right)\left(1-e^{-\delta}\right)^{-1}
$$

Então vemos facilmente que

$$
\begin{aligned}
X_{n} & =e^{-\epsilon_{n}^{(m)}(a-\delta)(n-m)} G_{\delta}(n, m) \\
& =e^{-(a-\delta)|n-m|} G_{\delta}(n, m)
\end{aligned}
$$

é uma solução limitada de (1.18) satisfazendo $P_{0} X_{0}=0$ para $J=Z^{+}$e $m \geq 1$ e assim por diante. Logo pela unicidade

$$
G(n, m)=e^{-(a-\delta)|n-m|} G_{\delta}(n, m)
$$

Portanto, temos a estimativa

$$
|G(n, m)| \leq 2 K\left(1+\epsilon^{-\delta}\right)\left(1-e^{-\delta}\right)^{-1} e^{-(a-\delta)|n-m|}
$$

para $n, m \in J$.

Da equação (1.18) que $G(n, m)$ satisfaz, vemos que para $n \geq m$

$$
\begin{aligned}
G(n, m) & =\left(C_{n-1}+D_{n-1}\right) G(n-1, m) \\
& =\left(C_{n-1}+D_{n-1}\right) \ldots\left(C_{m}+D_{m}\right) G(m, m)
\end{aligned}
$$

Se tomarmos $n=m-1 \mathrm{em}(1.18) \operatorname{com} X_{n}=G(n, m)$, obtemos

$$
G(m, m)=\left(C_{m-1}+D_{m-1}\right) G(m-1, m)+I
$$

Logo, para $n<m$

$$
\begin{aligned}
-(I-G(m, m)) & =\left(C_{m-1}+D_{m-1}\right) G(m-1, m) \\
& =\left(C_{m-1}+D_{m-1}\right) \ldots\left(C_{n}+D_{n}\right) G(n, m)
\end{aligned}
$$

Portanto, se denotarmos por $\Psi(n, m)$ a matriz de transição para a equação (1.17) e escrevermos $Q_{m}=G(m, m)$, mostramos que

$$
G(n, m)=\left\{\begin{array}{cc}
\Psi(n, m) Q_{m} & \text { para } n \geq m \\
-\Psi(n, m)\left(I-Q_{m}\right) & \text { para } n<m
\end{array}\right.
$$

Por (1.20) tudo que resta a ser mostrado é que os $Q_{m}$ 's são projeções invariantes com respeito à equação (1.17). 
Para mostrar que $Q_{m}$ é uma projeção fixamos $m$ em $J$ e definimos para $n \in J$

$$
X_{n}=\left\{\begin{array}{cc}
\Psi(n, m) Q_{m}^{2}=G(n, m) Q_{m} & \text { para } n \geq m \\
-\Psi(n, m)\left(I-Q_{m}^{2}\right)=G(n, m)\left(I+Q_{m}\right) & \text { para } n<m
\end{array}\right.
$$

$X_{n}$ é limitada pois $G(n, m)$ o é. Também quando $n \neq m-1$ é fácil ver que

$$
X_{n+1}=\left(C_{n}+D_{n}\right) X_{n}
$$

Quando $n=m-1$,

$$
\begin{aligned}
X_{n+1}=X_{m}=Q_{m}^{2} & =-\left(I-Q_{m}^{2}\right)+I \\
& =-\left(C_{m-1}+D_{m-1}\right) \Psi(m-1, m)\left(I-Q_{m}^{2}\right)+I \\
& =\left(C_{m-1}+D_{m-1}\right) X_{m-1}+I
\end{aligned}
$$

Logo, $X_{n}$ é soluçâo de (1.18). Finalmente quando $J=\mathbf{Z}^{+}$e $m \geq 1, P_{0} X_{0}=0$ e assim por diante, pois $G(n, m)$ tem estas propriedades. Segue pela unicidade que $X_{n}=G(n, m)$ para $n \in J$. Tomando $n=m$, temos $Q_{m}^{2}=Q_{m}$. Assim $Q_{m}$ é na verdade uma projeção.

A invariância de $Q_{m}$ é provada por um tipo análogo de argumento. Fixe $m$ em $J\left(m \leq-1\right.$ se $\left.J=\mathbf{Z}^{-}\right)$e defina para $n \in J$

$$
X_{n}=\left\{\begin{array}{cc}
\Psi(n, m) Q_{m} \Psi(m, m+1) & \text { para } n>m \\
-\Psi(n, m)\left(I-Q_{m}\right) \Psi(m, m+1) & \text { para } n \leq m
\end{array}\right.
$$

$X_{n}=G(n, m) \Psi(m, m+1)$ para $n \neq m$ e logo é limitado. Quando $n \neq m$ é fácil ver que

$$
X_{n+1}=\left(C_{n}+D_{n}\right) X_{n}
$$

Quando $n=m$

$$
\begin{aligned}
X_{n+1}=X_{m+1} & =\Psi(m+1, m) Q_{m} \Psi(m, m+1) \\
& =\left(C_{m}+D_{m}\right) \Psi(m, m) Q_{m} \Psi(m, m+1) \\
& =\left(C_{m}+D_{m}\right)\left(-\Psi(m, m)\left(I-Q_{m}\right) \Psi(m, m+1)+\Psi(m, m+1)\right) \\
& =\left(C_{m}+D_{m}\right)\left(X_{m}+\Psi(m, m+1)\right) \\
& =\left(C_{m}+D_{m}\right) X_{m}+1
\end{aligned}
$$

Logo $X_{n}$ é uma soluçâo de (1.18) com $m+1$ no lugar de $m$. Finalmente, quando $J=\mathbf{Z}^{+}$e $m \geq 1, P_{0} X_{0}=0$ e quando $J=\mathbf{Z}^{-},\left(I-P_{0}\right) X_{0}=0$, pois $G(n, m)$ tem estas propriedades. Quando $J=Z^{+}$e $m=0$,

$$
P_{0} X_{0}=-P_{0}(I-G(0,0)) \Psi(0,1)=0
$$


pois $P_{0} G(0,0)=P_{0}$. Assim, pela unicidade, $X_{n}=G(n, m+1)$ para $n, m \in J$ ( $m \leq-1$ se $\left.J \in \mathbf{Z}^{-}\right)$. Em particular, tomando $n=m+1$, temos

$$
\Psi(m+1, m) Q_{m} \Psi(m, m+1)=G(m+1, m+1)=Q_{m+1}
$$

Isto é,

$$
\left(C_{m}+D_{m}\right) Q_{m}=Q_{m+1}\left(C_{m}+D_{m}\right)
$$

Logo, as projeções $Q_{m}$ são invariantes com respeito à equação (1.17) e a prova está concluída.

Observação 1.3 Podemos obter também uma estimativa para $\left|Q_{n}-P_{n}\right|$ no seguinte sentido: a funçâo de Green para o sistema não pertubado (1.1) é definido por

$$
G_{0}(n, m)=\left\{\begin{array}{cc}
\Phi(n, m) P_{m} & \text { para } n \geq m \\
-\Phi(n, m)\left(I-P_{m}\right) & \text { para } n<m
\end{array}\right.
$$

e é, para cada m, a única solução limitada da equação

$$
X_{n+1}=C_{n} X_{n}+H_{n}^{(m)}
$$

tal que $P_{0} X_{0}=0$ quando $J=\mathrm{Z}^{+}$e $m \geq 1$ e assim por diante. Logo, $W_{n}=G(n, m)-G_{0}(n, m)$ é a única solução limitada de

$$
W_{n+1}=C_{n} W_{n}+D_{n} W_{n}+D_{n} G_{0}(n, m)
$$

tal que $P_{0} W_{0}=0$ quando $J=\mathrm{Z}^{+} \epsilon\left(I-P_{0}\right) W_{0}=0$ quando $J=\mathbf{Z}^{-}$. Então pela Proposiçâo 1.4 e Observação 1.2, temos a estimativa

$$
\left|W_{n}\right| \leq 2 K\left(1+\epsilon^{-\alpha}\right)\left(1-e^{-\alpha}\right)^{-1} \sup _{n \in J}\left|D_{n}\right| K
$$

Tome $n=m$ e temos para todo $m \in m J$,

$$
\left|Q_{m}-P_{m}\right| \leq 2 K^{2}\left(1+\epsilon^{-\alpha}\right)\left(1-e^{-\alpha}\right)^{-1} \sup _{n \in J}\left|D_{n}\right| .
$$




\section{Capítulo 2}

\section{Conjuntos Hiperbólicos e o Shadowing Lemma}

O resultado principal deste capítulo é o shadowing lemma. A toda órbita de um difeomorfismo associamos uma equação diferença linear chamada equação variacional, e diremos que um conjunto invariante é hiperbólico se a equação variacional de cada órbita neste conjunto tem uma dicotomia exponencial. $O$ shadowing lemma, que é uma propriedade de conjuntos hiperbólicos, passa a ser uma consequencia de sistemas quasilinear provado no capítulo anterior.

Seja $f: \mathbf{R}^{p} \rightarrow \mathbf{R}^{p}$ um difeomorfismo $C^{1}$. Um subconjunto $S$ de $\mathbf{R}^{p}$ é invariante se $f(S)=S$.

Definição 2.1 Um conjunto invariante compacto $S \dot{e}$ hiperbólico se para cada $x$ em $S$ a 'equação variacional' ao longo da órbita $\left\{f^{n}(x)\right\}$

$$
u_{n+1}=D f\left(f^{n}(x)\right) u_{n}
$$

tem uma dicotomia exponencial em $\mathrm{Z}$ com constantes $K$, a e os postos das projeçôes $P_{n}(x)$ independentes de $x$.

Observação 2.1 Se denotarmos $C_{n}(x)=D f\left(f^{n}(x)\right)$, note que a matriz de transiçâo da equaçâo (2.1) é dada por

$$
\Phi(n, m)(x)=\left\{\begin{array}{cccc}
C_{n-1}(x) & \ldots & C_{m}(x) & \text { se } n>m \\
I & \ldots & & \text { se } n=m \\
C_{n}^{-1}(x) & \ldots & C_{m-1}^{-1}(x) & \text { se } n<m
\end{array}\right.
$$


Mas, para $n>m$

$$
\begin{aligned}
C_{n-1}(x) C_{n-2}(x) \ldots C_{m}(x) & =D f\left(f^{n-1}(x)\right) D f\left(f^{n-2}(x)\right) \ldots D f\left(f^{m}(x)\right) \\
& =D f^{n-m}\left(f^{m}(x)\right)
\end{aligned}
$$

temos também, para $n<m$, que

$$
\Phi(n, m)=[\Phi(m, n)]^{-1}=\left[D f^{m-n}\left(f^{n}(x)\right)\right]^{-1}=D f^{n-m}\left(f^{m}(x)\right)
$$

pois

$$
I=D I(y)=D\left(f^{-k} \circ f^{k}\right)(y)=D f^{-k}\left(f^{k}(y)\right) \circ D f^{k}(y)
$$

donde

$$
\left[D f^{k}(y)\right]^{-1}=D f^{-k}\left(f^{k}(y)\right)
$$

Portanto,

$$
\Phi(n, m)(x)=D f^{n-m}\left(f^{m}(x)\right)
$$

para todo $n, m \in J$.

A Proposição seguinte nos dá uma definição alternativa para conjuntos hiperbólicos.

Proposição 2.1 Seja $f: \mathbf{R}^{p} \rightarrow \mathbf{R}^{p}$ um difeomorfismo $C^{1}$ e $S$ um conjunto invariante compacto. Então $S$ é hiperbólico se, e somente se, existem constantes positivas $K$, a e uma funçâo matriz projeção $\mathcal{P}(x), x \in S$, de posto constante tal que para todo $x$ em $S$

$$
\begin{gathered}
\mathcal{P}(f(x)) D f(x)=D f(x) \mathcal{P}(x) \\
\left|D f^{n}(x) \mathcal{P}(x)\right| \leq K e^{-\alpha n} \quad(n \geq 0) \\
\left|D f^{n}(x)(I-\mathcal{P}(x))\right| \leq K e^{\alpha n} \quad(n \leq 0)
\end{gathered}
$$

\section{Prova:}

Note, pela observação acima, que a matriz de transiçào para a equação (2.1) é dada por

$$
\Phi(n, m)(x)=D f^{n-m}\left(f^{m}(x)\right)
$$

$(\Leftarrow)$ Defina

$$
P_{n}(x)=\mathcal{P}\left(f^{n}(x)\right)
$$


e mostremos que (2.1) tem uma dicotomia exponencial em $\mathbf{Z}$ com constantes $K, \alpha$ e projeçôes $P_{n}(x)$. $P_{n}(x)$ é invariante, pois

$$
\begin{aligned}
P_{n+1}(x) D f\left(f^{n}(x)\right) & =\mathcal{P}\left(f^{n+1}(x)\right) D f\left(f^{n}(x)\right) \\
& =D f\left(f^{n}(x)\right) \mathcal{P}\left(f^{n}(x)\right) \\
& =D f\left(f^{n}(x)\right) P_{n}(x)
\end{aligned}
$$

Também, para $n \geq m$, temos

$$
\left|\Phi(n, m)(x) P_{m}(x)\right|=\left|D f^{n-m}\left(f^{m}(x)\right) \mathcal{P}\left(f^{m}(x)\right)\right| \leq K e^{-\alpha(n-m)}
$$

e para $n \leq m$

$$
\left|\Phi(n, m)(x)\left(I-P_{m}(x)\right)\right|=\left|D f^{n-m}\left(f^{m}(x)\right)\left(I-\mathcal{P}\left(f^{m}(x)\right)\right)\right| \leq K e^{-\alpha(m-n)}
$$

onde usamos (2.3) e (2.4).

$\Rightarrow$ ) Sejam $K$, a e $P_{n}(x)$ como na Definição 2.1. Note primeiramente que para qualquer inteiro $m$ a equaçâo variacional

$$
u_{n+1}=D f\left(f^{n}\left(f^{m}(x)\right)\right) u_{n}
$$

tem uma dicotomia exponencial em $\mathbf{Z}$ com projeções $P_{n}\left(f^{m}(x)\right)$. Mas esta equação diferença também pode ser escrita como

$$
u_{n+1}=D f\left(f^{n+m}(x)\right) u_{n}
$$

da qual, por comparação com (2.1), tem uma dicotomia exponencial com projeçôes $P_{n+m}$. De fato, seja $u_{n+1}=\hat{C}_{n}(x) u_{n}$, onde $\tilde{C}_{n}(x)=D f\left(f^{n+m}(x)\right)$. Como $C_{n+m}(x)=D f\left(f^{n+m}(x)\right)$, tem-se que $\hat{C}_{n}(x)=C_{n+m}(x)$. A equação acima tem projeçôes $\left\{\hat{P}_{n}(x)\right\}$ cujo índice está associado ao de $\left\{\tilde{C}_{n}(x)\right\}$. Mas, $\tilde{C}_{n}(x)=C_{n+m}(x)$ cujo indice de $\left\{C_{n}(x)\right\}$ está associado ao de $\left\{P_{n}(x)\right\}$. Pela Definição 2.1, $\hat{P}_{n}(x)=P_{n}\left(f^{m}(x)\right)$. Da unicidade das projeções (Proposição 1.2 (iii)) segue que $P_{n+m}(x)=P_{n}\left(f^{m}(x)\right)$. Em particular, temos a identidade

$$
P_{n}(x)=P_{0}\left(f^{n}(x)\right)
$$

Defina

$$
\mathcal{P}(x)=P_{0}(x)
$$

Então $\mathcal{P}(x)$ é de posto constante e segue da identidade (2.5) que

$$
P_{n}(x)=\mathcal{P}\left(f^{n}(x)\right)
$$

Assim, para todo $x$

$$
\begin{aligned}
\mathcal{P}(f(x)) D f(x) & =P_{1}(x) D f\left(f^{0}(x)\right) \\
& =D f\left(f^{0}(x)\right) P_{0}(x) \\
& =D f(x) \mathcal{P}(x)
\end{aligned}
$$


Portanto, temos a propriedade de invariância (2.2). Também, para todo $x$ em $S$ e $n \geq 0$,

$$
\left|D f^{n}(x) \mathcal{P}(x)\right|=\left|\Phi(n, 0)(x) P_{0}(x)\right| \leq K e^{-\alpha n}
$$

e para $n \leq 0$,

$$
\left|D f^{n}(x)(I-\mathcal{P}(x))\right|=\left|\Phi(n, 0)(x)\left(I-P_{0}(x)\right)\right| \leq K e^{\alpha_{n}}
$$

Isto estabelece (2.3) e (2.4) e completa a prova da proposição .

Proposição 2.2 Seja $\mathcal{P}(x)$ como na Proposição 2.1. Então $\mathcal{P}(x)$ é contínua em $S$.

Prova:

Segue de (2.3) com $n=0$ que

$$
|\mathcal{P}(x)| \leq K
$$

para todo $x$ em $S$. Como $S$ é compacto, para mostrar que $\mathcal{P}$ é contínua é suficiente mostrar que a aplicaçâo $x \rightarrow \mathcal{P}(x)$ tem gráfico fechado. Tome $x_{k} \rightarrow x \mathrm{e}$ $\mathcal{P}\left(x_{k}\right) \rightarrow Q$. Temos que mostrar que $Q=\mathcal{P}(x)$. Mas de (2.3) e de (2.4), temos as desigualdades

$$
\begin{gathered}
\left|D f^{n-m}\left(f^{m}\left(x_{k}\right)\right) \mathcal{P}\left(f^{m}\left(x_{k}\right)\right)\right| \leq K e^{-\alpha(n-m)}(n \geq m) \\
\left|D f^{n-m}\left(f^{m}\left(x_{k}\right)\right)\left(I-\mathcal{P}\left(f^{m}\left(x_{k}\right)\right)\right)\right| \leq K e^{-\alpha(m-n)}(m \geq n)
\end{gathered}
$$

Também, por repetidas aplicaçôes de (2.2), obtemos

$$
\mathcal{P}\left(f^{n}\left(x_{k}\right)\right)=D f^{n}\left(x_{k}\right) \mathcal{P}\left(x_{k}\right) D f^{-n}\left(f^{n}\left(x_{k}\right)\right)
$$

Fazendo $k \rightarrow \infty$ em (2.6), (2.7) e (2.8) obtemos

$$
\begin{gathered}
\left|D f^{n-m}\left(f^{m}(x)\right) Q_{m}\right| \leq K \epsilon^{-\alpha(n-m)} \quad(n \geq m) \\
\left|D f^{n-m}\left(f^{m}(x)\right)\left(I-Q_{m}\right)\right| \leq K \epsilon^{-\alpha(m-n)} \quad(m \geq n)
\end{gathered}
$$

onde $Q_{n}=D f^{n}(x) Q D f^{-n}\left(f^{n}(x)\right)$. Isto significa que a equação diferença linear (2.1), que tem matriz de transiçâo $D f^{n-m}\left(f^{m}(x)\right)$, tem uma dicotomia exponencial em $\mathbf{Z}$ com projeçôes $Q_{n}$. Mas já sabemos que ela tem uma dicotomia exponencial com projeçôes $P_{n}(x)=\mathcal{P}\left(f^{n}(x)\right)$. Logo pela unicidade (Proposição 1.2 (iii)) $Q_{n}=\mathcal{P}\left(f^{n}(x)\right)$ para todo $n$. Em particular, tomando $n=0$, temos $Q=\mathcal{P}(x)$. 
Definição 2.2 Uma sequência duplamente infinita (ou bisequência) em $\mathbf{R}^{p}$ é uma $\delta$ pseudo-órbita de um difeomorfismo $C^{1} f: \mathbf{R}^{p} \rightarrow \mathbf{R}^{p}$ se para todo inteiro $n$

$$
\left|y_{n+1}-f\left(y_{n}\right)\right| \leq \delta
$$

Uma órbita $\left\{f^{n}(x)\right\}$ e-shadow(€-sombreia) uma $\delta$ pseudo-órbita se para todo inteiro $n$

$$
\left|f^{n}(x)-y_{n}\right| \leq \epsilon
$$

Enunciamos e provamos agora o 'shadowing lemma'.

Teorema 2.1 (shadowing lemma) Seja $S$ um conjunto hiperbólico compacto para o difeomorfismo $C^{1} f: \mathbf{R}^{p} \rightarrow \mathbf{R}^{p}$. Então dado $\epsilon>0$ suficientemente pequeno, existe $\delta>0$ tal que toda $\delta$ pseudo-órbita em $S$ tem uma única órbita que a €-shadow.

\section{Prova:}

Sejam $K, \alpha$ e $\mathcal{P}(x)$ como na Definição 2.1 e na Proposição 2.1. Denote por $B$ uma bola fechada limitada contendo $S$ e defina

$$
M=\sup _{x \in B}|D f(x)|
$$

Denote por $w_{1}($.$) o módulo de continuidade para D f(x)$ com $x$ em $B$. Isto é, se $\Delta>0$,

$$
w_{1}(\Delta)=\sup \{|D f(x)-D f(y)|: x, y \in B,|x-y| \leq \Delta\}
$$

Pela Proposição 2.2, $\mathcal{P}(x)$ é contínua no conjunto $S$. Denote por $w_{2}($.$) seu módulo$ de continuidade em $S$.

Temos que resolver a equação diferença não linear

$$
x_{n+1}=f\left(x_{n}\right) \quad(n \in \mathbf{Z})
$$

para uma sequência $\left\{x_{n}\right\}$ tal que $\left|x_{n}-y_{n}\right| \leq \epsilon$ para todo $n$. Escreva

$$
x_{n}=y_{n}+z_{n}
$$

Entâo $\left\{z_{n}\right\}$ é uma solução de

$$
z_{n+1}=C_{n} z_{n}+h_{n}+g_{n}\left(z_{n}\right)
$$


onde

$$
\begin{gathered}
C_{n}=D f\left(y_{n}\right) \\
g_{n}(z)=f\left(y_{n}+z\right)-f\left(y_{n}\right)-D f\left(y_{n}\right) z \\
h_{n}=f\left(y_{n}\right)-y_{n+1}
\end{gathered}
$$

Defina

$$
\begin{aligned}
P_{n} & =\mathcal{P}\left(y_{n}\right) \\
Q_{n+1} & =\mathcal{P}\left(f\left(y_{n}\right)\right)
\end{aligned}
$$

para $n \in \mathbf{Z}$. Então segue de (2.2) que, para todo $n$,

$$
Q_{n+1} C_{n}=C_{n} P_{n}
$$

Agora defina

$$
D_{n}=P_{n+1} C_{n} P_{n}+\left(I-P_{n+1}\right) C_{n}\left(I-P_{n}\right)
$$

Pode ser facilmente verificado que

$$
P_{n+1} D_{n}=D_{n} P_{n}
$$

Também,

$$
\begin{aligned}
\left|C_{n}-D_{n}\right| & =\left|\left(I-P_{n+1}\right) C_{n} P_{n}+P_{n+1} C_{n}\left(I-P_{n}\right)\right| \\
& =\left|\left(I-P_{n+1}\right) Q_{n+1} C_{n}+P_{n+1}\left(I-Q_{n+1}\right) C_{n}\right| \\
& =\left|\left[\left(Q_{n+1}-P_{n+1}\right) Q_{n+1}+P_{n+1}\left(P_{n+1}-Q_{n+1}\right)\right] C_{n}\right| \\
& =\mid\left[\left(Q_{n+1}-P_{n+1}\right)\left(Q_{n+1}-P_{n+1}\right] C_{n} \mid\right. \\
& \leq\left\{\left|Q_{n+1}\right|+\left|P_{n+1}\right|\right\}\left|C_{n}\right|\left|Q_{n+1}-P_{n+1}\right| \\
& \leq 2 K M w_{2}(\delta)
\end{aligned}
$$

Portanto, para todo $n$,

$$
\left|C_{n}-D_{n}\right| \leq 2 K M w_{2}(\delta)
$$

Reescrevemos (2.9) como

$$
z_{n+1}=D_{n} z_{n}+h_{n}+\left[\left(C_{n}-D_{n}\right) z_{n}+g_{n}\left(z_{n}\right)\right]
$$

e queremos aplicar a Proposição 1.4. Para fazer isto, devemos primeiro mostrar que a equação linear

$$
z_{n+1}=D_{n} z_{n}
$$

tem uma dicotomia exponencial em Z . Mostraremos, na verdade, que a equação tem uma dicotomia exponencial com projeções $P_{n}$. A invariância de $P_{n}$ segue da 
definição de $D_{n}$ e da igualdade $P_{n+1} D_{n}=D_{n} P_{n}$.

Usando a desigualdade

$$
\begin{aligned}
\left|y_{n+k+1}-f^{k+1}\left(y_{n}\right)\right| & \leq\left|y_{n+k+1}-f\left(y_{n+k}\right)\right|+\left|f\left(y_{n+k}\right)-f\left(f^{k}\left(y_{n}\right)\right)\right| \\
& \leq \delta+M\left|y_{n+k}-f^{k}\left(y_{n}\right)\right|
\end{aligned}
$$

podemos mostrar, por indução em $k$, que

$$
\left|y_{n+k}-f^{k}\left(y_{n}\right)\right| \leq\left(1+M+\ldots+M^{k-1}\right) \delta
$$

para todo $n$ e $k \geq 1$.

Denote por $\Psi(n, m)$ a matriz de transição para (2.13). Fixe $n$ e defina

$$
d_{k}=\left|\Psi(n+k, n) P_{n}-D f^{k}\left(y_{n}\right) P_{n}\right|,
$$

onde $k \geq 1$. Escolha o menor inteiro positivo $N$ tal que

$$
K e^{-\alpha N} \leq 1 / 2
$$

Nosso objetivo é estimar $d_{N}$. Façamos isto obtendo uma estimativa para $d_{k+1}$ em termos de $d_{k}$ para $1 \leq k \leq N-1$. Primeiro devemos supor que

$$
\left(1+M+\ldots+M^{N-1}\right) \delta \leq \operatorname{dist}\left(S, B^{c}\right) .
$$

Isto assegura que $f^{k}\left(y_{n}\right) \in B$ para todo $n$ e $1 \leq k \leq N$, usando (2.14). Entâo

$$
\begin{aligned}
d_{k+1}= & \left|\Psi(n+k+1, n) P_{n}-D f^{k+1}\left(y_{n}\right) P_{n}\right| \\
\leq & \left|\Psi(n+k+1, n) P_{n}-D f\left(f^{k}\left(y_{n}\right)\right) \Psi(n+k, n) P_{n}\right| \\
& +\left|D f\left(f^{k}\left(y_{n}\right)\right) \Psi(n+k, n) P_{n}-D f\left(f^{k}\left(y_{n}\right)\right) D f^{k}\left(y_{n}\right) P_{n}\right| \\
\leq & \left|D_{n+k}-D f\left(f^{k}\left(y_{n}\right)\right)\right|\left|\Psi(n+k, n) P_{n}\right| \\
& +\left|D f\left(f^{k}\left(y_{n}\right)\right)\right|\left|\Psi(n+k, n) P_{n}-D f^{k}\left(y_{n}\right) P_{n}\right| \\
\leq & \left\{\left|D_{n+k}-C_{n+k}\right|+\left|D f\left(y_{n+k}\right)-D f\left(f^{k}\left(y_{n}\right)\right)\right|\right\}\left|\Psi(n+k, n) P_{n}\right| \\
& +\left|D f\left(f^{k}\left(y_{n}\right)\right)\right|\left|\Psi(n+k, n) P_{n}-D f^{k}\left(y_{n}\right) P_{n}\right| \\
\leq & \left\{2 K M w_{2}(\delta)+w_{1}\left(\left(1+M+\ldots+M^{k-1}\right) \delta\right)\right\} 2^{k} M^{k} K^{2 k} K+M d_{k}
\end{aligned}
$$

onde usamos (2.11), (2.14) e o fato que $2 M K^{2}$ é um limitante superior para $\left|D_{n}\right|$.

$$
\begin{aligned}
\left|D_{n}\right| & =\left|P_{n+1} C_{n} P_{n}+\left(I-P_{n+1}\right) C_{n}\left(I-P_{n+1}\right)\right| \\
& \leq\left|P_{n+1} C_{n} P_{n}\right|+\left|\left(I-P_{n+1}\right) C_{n}\left(I-P_{n+1}\right)\right| \\
& \leq K\left|C_{n}\right| K+K\left|C_{n}\right| K \\
& =2 K^{2}\left|D f\left(y_{n}\right)\right| \leq 2 M K^{2} .
\end{aligned}
$$


Portanto, para $1 \leq k \leq N-1$,

$$
d_{k+1} \leq w_{3}(\delta)+M d_{k}
$$

onde

$$
w_{3}(\delta)=\sup _{1 \leq k \leq N-1} 2^{k} M^{k} K^{2 k+1}\left\{2 K M w_{2}(\delta)+w_{1}\left(\left(1+M+\ldots+M^{k-1}\right) \delta\right)\right\}
$$

Segue por induçào em $k$ que

$$
d_{N} \leq\left(1+M+\ldots+M^{N-2}\right) w_{3}(\delta)+M^{N-1} d_{1}
$$

Mas,

$$
d_{1}=\left|\left(D_{n}-C_{n}\right) P_{n}\right| \leq 2 K^{2} M w_{2}(\delta)
$$

Logo,

$$
d_{N} \leq\left(1+M+\ldots+M^{N-2}\right) w_{3}(\delta)+2 K^{2} M^{N} w_{2}(\delta) \leq 1 / 4
$$

se $\delta$ é suficientemente pequeno.

Então segue que para todo $n$,

$$
\begin{aligned}
\left|\Psi(n+N, n) P_{n}\right| & \leq d_{N}+\left|D f^{N}\left(y_{n}\right) P_{n}\right| \\
& =d_{N}+\left|D f^{N}\left(y_{n}\right) \mathcal{P}\left(y_{n}\right)\right| \\
& \leq d_{N}+K e^{-\alpha N} \leq 1 / 4+1 / 2
\end{aligned}
$$

Assim, para todo $n$,

$$
\left|\Psi(n+N, n) P_{n}\right| \leq 3 / 4
$$

Desejamos extender esta última desigualdade a uma para $\left|\Psi(n, m) P_{n}\right|$ onde $n \geq m$. Existe um único inteiro nâo negativo $k$ tal que

$$
m+k N \leq n<m+(k+1) N .
$$

Então,

$$
\begin{aligned}
& \left|\Psi(n, m) P_{m}\right| \\
& =\left|\Psi(n, m+k N) \Psi(m+k N, m+(k+1) N) \ldots \Psi(m+N, m) P_{m}\right| \\
& =\left|\Psi(n, m+k N) \Psi(m+k N, m+(k+1) N) P_{m+(k-1) N} \ldots \Psi(m+N, m) P_{m}\right| \\
& \leq|\Psi(n, m+k N)|\left|\Psi(m+k N, m+(k+1) N) P_{m+(k-1) N}\right| \ldots\left|\Psi(m+N, m) P_{m}\right| \\
& \leq\left(2 M K^{2}\right)^{N-1}\left(\frac{3}{4}\right)^{k} \leq\left(2 M K^{2}\right)^{N-1}\left(\frac{3}{4}\right)^{\frac{n-m}{N}-1} .
\end{aligned}
$$

Logo, para $n \geq m$,

$$
\left|\Psi(n, m) P_{m}\right| \leq K_{1} \epsilon^{-\alpha_{1}(n-m)}
$$


onde $K_{1}=\frac{4}{3}\left(2 M K^{2}\right)^{N-1}$ e $\alpha_{1}=\frac{\log \frac{4}{3}}{N}$.

Analogamente podemos mostrar que para $m \geq n$

$$
\left|\Psi(n, m)\left(I-P_{m}\right)\right| \leq K_{1} e^{-\alpha_{1}(m-n)}
$$

Portanto, mostramos que quando $\delta>0$ é tào pequeno que (2.15) e (2.16) sâo satisfeitos, a equação linear (2.13) tem uma dicotomia exponencial em $\mathbf{Z}$ com constantes $K_{1}, a_{1}$ e projeções $P_{n}$.

Agora aplicamos a Proposição 1.4 à equação (2.12) para provar a existência de uma única solução $z_{n}$ tal que $\left|z_{n}\right| \leq \epsilon$ para todo $n$. O termo não linear em (2.12) tem constante de Lipschitz

$$
\left|C_{n}-D_{n}\right|+w_{1}(\epsilon)
$$

desde que restrinjamos $z$ a $\left|z_{n}\right| \leq \epsilon$ e assumamos $\epsilon \leq \operatorname{dist}\left(S, B^{c}\right)$. Logo a condição sobre a constante de Lipschitz na Proposição 1.4 será satisfeita desde que

$$
\begin{gathered}
4 K_{1}^{\prime}\left(1+e^{-\alpha_{1}}\right)\left(1-e^{-\alpha_{1}}\right)^{-1} 2 K M w_{2}(\delta) \leq 1 \\
4 K_{1}\left(1+e^{-\alpha_{1}}\right)\left(1-e^{-\alpha_{1}}\right)^{-1} w_{1}(\epsilon) \leq 1
\end{gathered}
$$

onde usamos (2.11). Também a condição sobre $h_{n}$ será satisfeita desde que

$$
2 K_{1}\left(1+\epsilon^{-\alpha_{1}}\right)\left(1-\epsilon^{-\alpha_{1}}\right)^{-1} \delta \leq \epsilon
$$

Portanto, se $\epsilon>0$ satisfaz $\epsilon \leq \operatorname{dist}\left(S, B^{c}\right)$ e (2.18) e $\delta>0$ é escolhido tão pequeno que (2.15), (2.16), (2.17) e (2.19) são sạtisfeitas, a equação diferença (2.9) tem uma única solução $z_{n}$ tal que $\left|z_{n}\right| \leq \epsilon$ para todo $n$.

Observação 2.2 A equaçâo variacional da shadowing órbita $x_{n}$ também tem uma dicotomia exponencial em $\mathrm{Z}$ desde que $\epsilon \epsilon \delta$ sejam suficientemente pequenos. A equaçâo variacional é, na verdade,

$$
u_{n+1}=D_{n} u_{n}+\left\{C_{n}-D_{n}+D g_{n}\left(z_{n}\right)\right\} u_{n}
$$

Sabemos que a equaçâo (2.13) tem uma dicotomia exponencial em $\mathbf{Z}$ com constantes $K_{1}, a_{1}$. Logo, pela Proposiçâo 1.5 a equação (2.20) também terá uma dicotomia em $\mathrm{Z}$ com projeções de mesmo posto desde que

$$
\sup \left\{\left|C_{n}-D_{n}\right|+\left|D g_{n}\left(z_{n}\right)\right|\right\} \leq 2 K M w_{2}(\delta)+w_{1}(\epsilon)
$$

seja suficientemente pequeno, e isto pode ser alcançado fazendo $\delta$ e $\epsilon$ pequenos. 


\section{Capítulo 3}

\section{Comportamento Caótico na vizinhança de um ponto homoclínico transversal: O Teorema de Smale}

Neste capitulo o shadowing lemma visto no capitulo anterior será usado para provar o Teorema de Smale. Os pontos homoclínicos transversais são definidos e o conceito de transversalidade é estudado puramente em termos da equação variacional da órbita do ponto homoclínico transversal.

Seja $f: \mathbf{R}^{p} \rightarrow \mathbf{R}^{p}$ um difeomorfismo $C^{\mathbf{l}}$.

Definição $3.1 x_{0} \in R^{p}$ é um ponto fixo hiperbólico de $f$ se $f\left(x_{0}\right)=x_{0}$ e os autovalores de $D f\left(x_{0}\right)$ estão fora do círculo unitário.

Seja $x_{0}$ um ponto fixo hiperbólico de $f$ e denote por $P$ a projeção com imagem a soma dos autoespaços generalizados de $D f\left(x_{0}\right)$ correspondentes aos autovalores dentro do círculo unitário, e com núcleo a soma dos autoespaços generalizados correspondentes aos autovalores fora do círculo unitário. Então $D f\left(x_{0}\right)$ comuta com $P$ e existem constantes positivas $K$, a tal que

$$
\begin{gathered}
\left|D f^{n}\left(x_{0}\right) P\right| \leq K \epsilon^{-\alpha \dot{n}} \quad(n \geq 0) \\
\left|D f^{n}\left(x_{0}\right)(I-P)\right| \leq K e^{\alpha n}(n \leq 0)
\end{gathered}
$$

Isto significa que a equaçâo variacional ao longo da órbita $\left\{x_{0}\right\}$

$$
u_{n+1}=D f\left(x_{0}\right) u_{n}
$$


tem uma dicotomia exponencial em $\mathbf{Z}$ com projeçào constante $P$. Logo, se $x_{0}$ é um ponto fixo hiperbólico, o conjunto $\left\{x_{0}\right\}$ é um conjunto hiperbólico no sentido da Definição 2.1.

Definição $3.2 y_{0} \in \mathbf{R}^{p} \dot{e}$ um ponto homoclínico com respeito ao ponto fixo hiperbólico $x_{0}$ se $y_{0} \neq x_{0}$ e $f^{n}\left(y_{0}\right) \rightarrow x_{0}$ quando $|n| \rightarrow \infty$.

A proposição abaixo analisa a equação variacional ao longo da órbita de um ponto homoclínico.

Proposição 3.1 Seja yo um ponto homoclínico com respeito a um ponto fixo hiperbólico $x_{0}$ de um difeomorfismo $C^{1} f: \mathbf{R}^{p} \rightarrow \mathbf{R}^{p}$. Entâo a equação variacional

$$
u_{n+1}=D f\left(f^{n}\left(y_{0}\right)\right) u_{n}
$$

tem uma dicotomia exponencial em $\mathbf{Z}^{+}$e em $\mathbf{Z}^{-}$com o posto da projeção, em ambos os casos, igual ao número de autovalores de $D f\left(x_{0}\right)$ dentro do círculo unitário.

\section{Prova:}

A equaçâo (3.2) tem uma dicotomia exponencial em $\mathbf{Z}$ com projeções constantes $P$, o posto de $P$ sendo igual ao número de autovalores de $D f\left(x_{0}\right)$ dentro do círculo unitário.

Para $q \geq 0$ fixado considere a equação

$$
u_{n+1}=D f\left(f^{n+q}\left(y_{0}\right)\right) u_{n}=\left[D f\left(x_{0}\right)+D f\left(f^{n+q}\left(y_{0}\right)\right)-D f\left(x_{0}\right)\right] u_{n}
$$

Podemos fazer $\left|D f\left(f^{n+q}\left(y_{0}\right)\right) u_{n}+D f\left(x_{0}\right)\right|$ uniformemente pequeno para $n \geq 0$ escolhendo $q$ suficientemente grande. Logo, segue da Proposição 1.5 que se $q$ é suficientemente grande, a equaçào (3.4) tem uma dicotomia exponencial em $\mathbf{Z}^{+}$ com projeçôes com mesmo posto que $P$.

Afirmação : A equação (3.3) tem uma dicotomia exponencial em $[q, \infty)$. De fato, sejam $C_{n}=D f\left(f^{n}\left(y_{0}\right)\right) \operatorname{com} \Phi(n, m)=D f^{n-m}\left(f^{m}\left(y_{0}\right)\right)$ a matriz de transição da equação (3.3) e $\hat{C}_{n}=D f\left(f^{n+q}\left(y_{0}\right)\right)$ com $\tilde{\Phi}(n, m)=D f^{n-m}\left(f^{m+q}\left(y_{0}\right)\right)$ a matriz de transição da equação (3.4). Então, $\tilde{C}_{n}=C_{n+q}$ e $\tilde{P}_{n}=P_{n+q}$, logo

$$
\hat{\Phi}(n, m)=\hat{C}_{n-1} \ldots \hat{C}_{m}=C_{n-1+q} \ldots C_{m+q}=\Phi(n+q, m+q)
$$


Se $\tilde{n}=n+q$ e $\dot{m}=m+q$, então $\hat{n}, \hat{m} \in[q, \infty)$, logo $\tilde{C}_{n}=C_{\hat{n}}, \dot{P}_{n}=P_{\tilde{n}}$ e $\tilde{\Phi}(n, m)=\Phi(\tilde{n}, \hat{m})$.

Assim,

$$
\begin{gathered}
P_{\tilde{n}+1} C_{\tilde{n}}=C_{\tilde{n}} P_{\tilde{n}} \\
\left|\Phi(\tilde{n}, \tilde{m}) P_{\dot{m}}\right| \leq K e^{-\alpha(\tilde{n}-\dot{m})} \quad(\hat{m} \leq \tilde{n}) \\
\left|\Phi(\tilde{n}, \tilde{m})\left(I-P_{\tilde{m}}\right)\right| \leq K e^{-\alpha(\tilde{m}-\tilde{n})} \quad(\tilde{n} \leq \tilde{m})
\end{gathered}
$$

logo (3.3) tem um dicotomia exponencial em $[q, \infty]$ e portanto, pela Proposição $1.1 \mathrm{em} \mathrm{Z}^{+}$, com projeçôes com mesmo posto de $P$.

Definição 3.3 Seja $y_{0}$ um ponto homoclínico com respeito a um ponto fixo hiperbólico $x_{0}$ de um difeomorfismo $C^{1} f: \mathbf{R}^{p} \rightarrow \mathbf{R}^{p}$. $y_{0} \dot{\epsilon}$ um ponto homoclínico transversal se a única soluçâo limitada em $\mathbf{Z}$ da equaçâo variacional (3.3) for a solução trivial.

Proposição 3.2 Seja $y_{0}$ um ponto homoclínico transversal com respeito a um ponto fixo hiperbólico $x_{0}$ de um difeomorfismo $C^{1} f: \mathbf{R}^{p} \rightarrow \mathbf{R}^{p}$. Então o conjunto

$$
\left\{x_{0}\right\} \cup\left\{f^{n}\left(y_{0}\right): n \in \mathbf{Z}\right\}
$$

é um conjunto hiperbólico.

\section{Prova:}

Pela Proposição 3.1 a equação variacional (3.3) tem uma dicotomia exponencial em $\mathrm{Z}^{+}$e em $\mathbf{Z}^{-}$com projeções do mesmo posto. Como $y_{0}$ é transversal, (3.3) não tem soluçâo limitada em Z. Segue pela Proposição 1.3 que (3.3) tem um ${ }^{*}$ dicotomia exponencial em $\mathbf{Z}$. Se $q$ é um inteiro fixo, pela comparação da matriz de transição para a equaçâo

$$
u_{n+1}=D f\left(f^{n}\left(f^{q}\left(y_{0}\right)\right)\right) u_{n}=D f\left(f^{n+q}\left(y_{0}\right)\right) u_{n}
$$

com a matriz de transiçào para (3.3), vemos que (3.5) tem um dicotomia exponencial em $\mathbf{Z}$ com as mesmas constantes e projeçôes com o mesmo posto de (3.3). Além disso, como $x_{0}$ é um ponto fixo hiperbólico sua equação variacional (3.2) tem uma dicotomia exponencial em $\mathbf{Z}$ com projeçôes tendo posto igual ao número de autovalores de $D f\left(x_{0}\right)$ dentro do círculo unitário. Como as projeções para (3.5) também tem este número como posto, e como existem apenas duas escolhas posssiveis para as constantes, segue da Definiçâo 2.1 que o conjunto 
$\left\{x_{0}\right\} \cup\left\{f^{n}\left(y_{0}\right): n \in \mathbf{Z}\right\}$, que é claramente compacto e invariante, é um conjunto hiperbólico.

Novamente, seja $y_{0}$ um ponto homoclínico transversal com respeito a um ponto fixo hiperbólico $x_{0}$ de um difeomorfismo $f: \mathbf{R}^{p} \rightarrow \mathbf{R}^{p}$. Entâo sabemos que o conjunto

$$
S=\left\{x_{0}\right\} \cup\left\{f^{n}\left(y_{0}\right): n \in \mathbf{Z}\right\}
$$

é hiperbólico. Nosso objetivo agora é usar o shadowing lemma para mostrar que a dinâmica de $f$ próxima a $S$ é caótica, isto é, que existe um conjunto invariante compacto no qual a ação de algum iterado do difeomorfismo é topologicamente conjugado à ação do shift de Bernoulli.

Definição 3.4 Seja $S$ como em (3.6). Um segmento de órbita de comprimento lé um sequência finita de l pontos da forma

$$
\left\{x_{0}, x_{0}, \ldots, x_{0}\right\}
$$

ou da forma

$$
\left\{f^{q+1}\left(y_{0}\right), f^{q+2}\left(y_{0}\right), \ldots, f^{q+l}\left(y_{0}\right)\right\}
$$

Considere as bisequências

$$
\ldots, C_{-1}, C_{0}, C_{1}, \ldots
$$

de segmentos de órbita. Arranjamos em sequência os pontos nos segmentos consecutivamente para formar um bisequência de pontos.

Pelo shadowing lemma (Teorema 2.1), dado $\epsilon>0$ suficientemente pequeno existe $\delta>0$ tal que qualquer $\delta$ pseudo-órbita em $S$ tem uma única $\epsilon$-shadowing órbita (esta última órbita pode não estar em $S$ ). Fixe um tal $\epsilon$ e o correpondente $\delta$ e considere as bisequências formadas de segmentos de órbita com a propriedade que o ponto extremo esquerdo e a imagem por $f$ do ponto extremo direito estão na bola de rajo $\delta / 2$ centrada em $x_{0}$ (Esta condição é sempre satisfeita quando o segmento de órbita contém apenas o ponto $x_{0}$ e nos outros casos podem ser satisfeitos escolhendo $q$ e $l$ suficientemente grandes). Então cada tal bisequência tem uma única $\epsilon$-shadowing órbita. Assim o que obtemos são órbitas que flutuam entre permanecer próxima a $x_{0}$ e seguir em torno da órbita de $y_{0}$, de modo aleatório. 
Definiçāo 3.5 Denote por $I_{m}(m \geq 2)$ o conjunto das bisequências

$$
a=\left(\ldots, a_{-1}, a_{0}, a_{1}, a_{2}, \ldots\right)
$$

onde $a_{i} \in\{1,2, \ldots, m\}$. Portanto $I_{m}=\{1,2, \ldots, m\}^{\mathrm{Z}}$. Damos ao conjunto $\{1,2, \ldots, m\}$ a topologia discreta e a $I_{m}$ a topologia produto. $O$ shift de Bernoulli $\beta: I_{m} \rightarrow I_{m}$ é o homeomorfismo definido por

$$
[\beta(a)]_{i}=a_{i+1}
$$

Teorema 3.1 (Teorema de Smale) Seja y um ponto homoclínico transversal com respeito a um ponto fixo hiperbólico $x_{0}$ de um difeomorfismo $C_{1} f: \mathbf{R}^{p} \rightarrow \mathbf{R}^{p}$. Então existe um inteiro $l$ e um homeomorfismo $\phi$ de $\mathcal{I}_{m}$ em um subconjunto compacto de $\mathbf{R}^{p}$ que $\dot{\epsilon}$ invariante por $f^{l}$, tal que

$$
f^{l} \circ \phi=\phi \circ \beta
$$

isto é, a ação de $f^{\prime}$ em $\phi\left(\mathcal{I}_{m}\right)$ é conjugada à ação de $\beta$ em $\mathcal{I}_{m}$, onde $\beta$ é o shift de Bernoulli.

\section{Prova:}

Sejam $p_{1}, p_{2}, \ldots, p_{r}$, onde $r=m-1$ ou $m$, inteiros distintos. Escolha e fixe $\epsilon>0$ tão pequeno quanto é exigido quando o shadowing lemma é aplicado ao conjunto hiperbólico $S=\left\{x_{0}\right\} \cup\left\{f^{n}\left(y_{0}\right): n \in \mathbf{Z}\right\}$ e tal que

$$
\epsilon<\frac{1}{2} \min _{i \neq j}\left|f^{p_{i}}\left(y_{0}\right)-f^{p_{2}}\left(y_{0}\right)\right|
$$

Quando $r=m-1$ exija também que

$$
\epsilon<\frac{1}{2} \min \left|f^{p_{i}}\left(y_{0}\right)-x_{0}\right|
$$

Agora tome $\delta>0$ correspondente a $\epsilon$ como no shadowing lemma e escolha $N$ tal que para $i=1, \ldots, r$,

$$
\left|f^{n}\left(y_{0}\right)-x_{0}\right|<\delta / 2 \text { quando } n=N+p_{i}+1 \text { ou } p_{i}-N \text {. }
$$

Para $i=1, \ldots, r$, definimos o segmento de órbita

$$
C_{i}=\left\{f^{p_{i}-N}\left(y_{0}\right), \ldots, f^{p_{1}}\left(y_{0}\right), \ldots, f^{p_{i}+N}\left(y_{0}\right)\right\}
$$


e se $r=m-1$, definimos

$$
C_{m}=\left\{x_{0}, \ldots, x_{0}\right\}
$$

onde existem $2 N+1 x_{0}$ 's.

Seja $a=\left(\ldots, a_{-1}, a_{o}, a_{1}, \ldots\right)$ em $\mathcal{I}_{m}$. Então, como na discussão anterior à Definição 3.5, a bisequência formada pelo arranjamento em sequência dos segmentos de órbita

$$
\ldots, C_{a_{-1}}, C_{a_{0}}, C_{a_{1}}, \ldots
$$

consecutivamente é uma $\delta$ pseudo-órbita $e$, portanto, tem uma única $\epsilon$-shadowing órbita. Definimos $\phi(a)$ sendo o ponto nesta órbita que 'sombreia' o ponto médio do segmento de órbita $C_{a_{0}}$. Assim, se denotarmos por $z_{i j}(a) \circ j$-ésimo ponto de $C_{a_{i}}, \phi(a)$ é o único ponto tal que

$$
\left|f^{N+(i-1)(2 N+1)+j}(\phi(a))-z_{i j}(a)\right| \leq \epsilon
$$

para todo $i$ e $j=1, \ldots, 2 N+1$. Se trocarmos $i$ por $i+1$, obtemos

$$
\left|f^{N+i(2 N+1)+j}(\phi(a))-z_{i j}(a)\right| \leq \epsilon
$$

mas isto pode ser reescrito como

$$
\left|f^{N+(i-1)(2 N+1)+j}\left(f^{2 N+1}(\phi(a))\right)-z_{i j}(\beta(a))\right| \leq \epsilon
$$

pois $a_{i+1}=[\beta(a)]_{i}$. Esta última desigualdade ocorre para todo $i$ e $j=1, \ldots$, $2 N+1$. Por comparação com (3.9) vemos pela unicidade que isto significa que

$$
\phi(\beta(a))=f^{2 N+1}(\phi(a))
$$

isto ocorre para todo $a \in \mathcal{I}_{m}$ e portanto,

$$
\phi \circ \beta=f^{2 N+1} \circ \phi
$$

Isto também mostra que $\phi\left(\mathcal{I}_{m}\right)$ é invariante por $f^{2 N+1}$.

Agora, mostremos que $\phi$ é $1-1$. Se $a \neq a^{\prime}$ então $a_{i} \neq a_{i}^{\prime}$ para algum i. Então,

$$
\begin{aligned}
& \left|f^{N+(i-j)(2 N+1)+(N+1)}(\phi(a))-f^{N+(i-j)(2 N+1)+(N+1)}\left(\phi\left(a^{\prime}\right)\right)\right| \\
& \geq\left|z_{i, N+1}(a)-z_{i, N+1}\left(a^{\prime}\right)\right|-\left|f^{N+(i-j)(2 N+1)+(N+1)}(\phi(a))-z_{i, N+1}(a)\right| \\
& \quad-\left|f^{N+(i-j)(2 N+1)+(N+1)}\left(\phi\left(a^{\prime}\right)\right)-z_{i, N+1}\left(a^{\prime}\right)\right| \\
& \geq\left|z_{i, N+1}(a)-z_{i, N+1}\left(a^{\prime}\right)\right|-2 \epsilon>0
\end{aligned}
$$

Logo, $\phi(a) \neq \phi\left(a^{\prime}\right)$, como requerido. 
A seguir mostraremos que $\phi$ é contínua. Caso contrário, existiria um ponto $a$ em $I_{m}$, um número $\Delta>0$ e uma sequência $a^{(k)}$ em $I_{m}$ tal que para todo $k$,

$$
a_{i}^{(k)}=a_{i} \text { para }|i| \leq k
$$

mas,

$$
\left|\phi\left(a^{(k)}\right)-\phi(a)\right| \geq \Delta
$$

$\phi\left(\mathcal{I}_{m}\right)$ está na $\epsilon$-vizinhança de $S$ e, portanto, é certamente limitada. Logo, tomando uma subsequência, se necessário, podemos assumir sem perda de generalidade que $\phi\left(a^{(k)}\right) \rightarrow x$ (digamos). Então de (3.10), obtemos

$$
|x-\phi(a)| \geq \Delta
$$

Usando agora (3.9), temos

$$
\left|f^{N+(i-1)(2 N+1)+j}\left(\phi\left(a^{(k)}\right)\right)-z_{i j}\left(a^{(k)}\right)\right| \leq \epsilon
$$

para todo $k$, i e $j=1 \ldots, 2 N+1$. Mas $C_{a_{i}^{(k)}}=C_{a_{i}}$, se $|i| \leq k$. Logo, se fixarmos $i$ e tomarmos $k \rightarrow \infty$, obtemos

$$
\left|f^{N+(i-1)(2 N+1)+j}(x)-z_{i j}(a)\right| \leq \epsilon
$$

para $i$ e $j=1 \ldots, 2 N+1$. Pela unicidade, devemos ter $x=\phi(a)$ que é uma contradição .

Portanto, $\phi$ é 1-1 e contínua. Como $\mathcal{I}_{m}$ é Hausdorff compacto segue que $\phi\left(\mathcal{I}_{m}\right)$ é compacto e que $\phi$ é um homeomorfismo.

Observação 3.1 O teorєma foi provado com $l=2 N+1$. É evidente que não é necessário que l seja impar. Ele foi assim escolhido apenas por uma questâo de conveniência.

Observação 3.2 Podemos facilmente arranjar, como Smale fez, para que o ponto homoclínico transversal yo esteja em $\phi\left(\mathcal{I}_{m}\right)$. Além disso, como $\phi\left(\mathcal{I}_{m}\right) e ́$ $f^{2 N+1}$-invariante, todos os iterados de $y_{0}$ por $f^{2 N+1}$ estão em $\phi\left(\mathcal{I}_{m}\right)$, e como $\phi\left(\mathcal{I}_{m}\right)$ é fechado, segue que $x_{0}$ também está em $\phi\left(\mathcal{I}_{m}\right)$.

Para ver isto, considere $r=m-1$ e $p_{1}=0$ e escolha $\delta$ e $N$ tal que satisfaçam as condições adicionais

$$
\delta \leq 2 \epsilon
$$


e

$$
\left|f^{n}\left(y_{0}\right)-x_{0}\right|<\delta / 2 \text { quando } n>N \text { ou } n \leq-N
$$

Então, a pseudo-órbita correspondente a

$$
a=(\ldots, m, m, 1, m, m, \ldots)
$$

é formada dos segmentos de órbitas

$$
\ldots, C_{m}, C_{m}, C_{1}, C_{m}, C_{m}, \ldots
$$

onde

$$
C_{1}=\left\{f^{-N}\left(y_{0}\right), \ldots, y_{0}, \ldots, f^{N}\left(y_{0}\right)\right\}
$$

e

$$
C_{m}=\left\{x_{0}, \ldots, x_{0}, \ldots, x_{0}\right\}
$$

É claro que a órbita de $y_{0},\left\{f^{n}\left(y_{0}\right)\right\}$, $\epsilon$-shadow esta pseudo-órbita e que $y_{0}$ 'sombreia' o ponto médio de $C_{a_{0}}=C_{a_{1}}$. Portanto, $\phi(a)=y_{0}$ e $y_{0} \in \phi\left(\mathcal{I}_{m}\right)$, como afirmado. 


\section{Capítulo 4}

\section{Equivalência de Definições de Ponto Homoclínico Transversal}

O propósito principal deste capítulo é mostrar que a definição de ponto homoclínico transversal dado no capítulo 3 é equivalente à definição usual em termos de variedades estável e instável. Mostramos também que próximo a um ponto homoclínico transversal existem infinitos outros tais pontos, e que difeomorfismos próximos também devem ter pontos homoclinicos transversais.

Primeiro provaremos a existência de uma variedade estável de um ponto fixo hiperbólico.

Proposição 4.1 Seja $x_{0}$ um ponto fixo hiperbólico de um difeomorfismo $C^{1}$ $f: \mathbf{R}^{p} \rightarrow \mathbf{R}^{p}$. Entâo existem constantes $K, \alpha$ e uma projeção $P$ comutando com $D f\left(x_{0}\right)$ tal que as desigualdades (3.1) ocorrem. Considere $\Delta>0$ com a propriedade que

$$
\left|D f(x)-D f\left(x_{0}\right)\right| \leq \gamma=\frac{1}{2} K^{-1}\left(1+e^{-\alpha}\right)\left(1-\epsilon^{-\alpha}\right)
$$

quando $\left|x-x_{0}\right| \leq \Delta$. Entâo existe uma aplicação $C^{1}$

$$
v:\{\xi \in \mathcal{R}(P):|\xi|<\Delta / 4 K\} \rightarrow \mathbf{R}^{p}
$$

com as seguintes propriedades:

(i) $x=v(\xi)$ é o único $x$ tal que

$$
\begin{gathered}
P\left(x-x_{0}\right)=\xi \\
\left|f^{n}(x)-x_{0}\right| \leq \Delta \text { para todo } \xi \in \mathcal{R}(P),
\end{gathered}
$$


(ii) $\left|v(\xi)-x_{0}\right| \leq \Delta$

$|D v(\xi)| \leq 2 K$ para todo $\xi \in \mathcal{R}(P)$

(iii) $v(0)=x_{0}$

$$
D v(0)=P
$$

(iv) vé 1-1.

\section{Prova:}

Dado $\xi \in \mathcal{R}(P)$ com $|\xi|<\Delta / 4 K$, procuramos $x$ tal que as condições (4.2) sejam válidas. Defina

$$
f^{n}(x)=x_{0}+z_{n}
$$

Então $z_{n}$ é um solução da equação diferença não linear

$$
z_{n+1}=D f\left(x_{0}\right) z_{n}+g\left(z_{n}\right)
$$

onde

$$
g(z)=f\left(x_{0}+z\right)-f\left(x_{0}\right)-D f\left(x_{0}\right) z
$$

Queremos

$$
P z_{0}=\xi(=P \xi) \text { e }\left|z_{n}\right| \leq \Delta \text { para } n \geq 0
$$

Note que como

$$
|D g(z)|=\left|D f\left(x_{0}+z\right)-D f\left(x_{0}\right)\right|
$$

segue de (4.1) que

$$
\left|g\left(z_{1}\right)-g\left(z_{2}\right)\right| \leq \gamma\left|z_{1}-z_{2}\right|
$$

para $\left|z_{1}\right|,\left|z_{2}\right| \leq \Delta$. Além disso,

$$
g(0)=0
$$

Assim, todas as condiçôes da Proposição 1.4 (ii) estão satisfeitas e concluímos que existe uma única soluçào da equaçào (4.3) satisfazendo às condições (4.5). Denote esta solução por $z_{n}(\xi)$.

Note que quando $\xi=0, z_{n}=0$ para todo $n$ é uma solução satisfazendo às condiçôes (4.5). Logo pela unicidade

$$
z_{n}(0)=0 \quad \text { para } n \geq 0
$$

Agora, definimos

$$
v(\xi)=x_{0}+z_{0}(\xi)
$$


logo para $n \geq 0$

$$
f^{n}(v(\xi))=x_{0}+z_{n}(\xi)
$$

Entâo (i) e os primeiro itens em (ii), (iii) seguem imediatamente. A primeira condição em (4.5) dá a jdentidade

$$
P\left(v(\xi)-x_{0}\right)=\xi
$$

da qual a injetividade de $v$ segue imediatamente.

Falta provar a suavidade de $v$. Primeiro mostremos que $z_{n}(\xi)$ é Lipschitziana em $\xi$. Sejam $\xi_{1}, \xi_{2}$ no domínio de $v$. Então $w_{n}=z_{n}\left(\xi_{1}\right)-z_{n}\left(\xi_{2}\right)$ é uma solução da equação não homogênea

$$
w_{n+1}=D f\left(x_{0}\right)\left(w_{n}\right)+\left[g_{n}\left(z_{n}(\xi)\right)-g_{n}\left(z_{n}\left(\xi_{2}\right)\right)\right]
$$

que é limitada em $\mathbf{Z}^{+}$e satisfaz $P w_{0}=P\left(\xi_{1}-\xi_{2}\right)$. Segue do Lema $1.1(i i)$ e de (4.6) que

$$
\|w\| \leq K\left|\xi_{1}-\xi_{2}\right|+K\left(1+e^{-\alpha}\right)\left(1-e^{-\alpha}\right)^{-1} \gamma\|w\|
$$

onde

$$
\|w\|=\sup _{n \geq 0}\left|w_{n}\right|
$$

Assim,

$$
\|w\| \leq K\left|\xi_{1}-\xi_{2}\right|+\frac{1}{2}\|w\|
$$

logo,

$$
\|w\| \leq 2 K^{\prime}\left|\xi_{1}-\xi_{2}\right|
$$

Portanto, para $n \geq 0$

$$
\left|z_{n}\left(\xi_{1}\right)-z_{n}\left(\xi_{2}\right)\right| \leq 2 K\left|\xi_{1}-\xi_{2}\right|
$$

Agora considere a equação matricial

$$
Z_{n+1}=\left[D f\left(x_{0}\right)+D g\left(z_{n}(\xi)\right)\right] Z_{n}
$$

onde $\left|D g\left(z_{n}(\xi)\right)\right| \leq \gamma$. Pela Proposiçâo 1.4 (ii) e observaçâo 1.2 esta equação tem uma única solução $Z_{n}(\xi)$ que é limitada em $Z^{+}$e satisfaz

$$
P Z_{0}(\xi)=P .
$$

Mostremos que $Z_{n}(\xi)=D z_{n}(\xi)$. Para este fim considere

$$
w_{n}=z_{n}(\xi+h)-z_{n}(\xi)-Z_{n}(\xi) h
$$


onde $\xi, h \in \mathcal{R}(P) \operatorname{com}|\xi|<\Delta / 4 K,|h|<\Delta / 4 K-|\xi|$. Vemos que $w_{n}$ é uma solução da equação

$$
w_{n+1}=D f\left(x_{0}\right) w_{n}+\left[g\left(z_{n}(\xi+h)\right)-g\left(z_{n}(\xi)\right)-D g\left(z_{n}(\xi)\right) Z_{n}(\xi) h\right]
$$

que é limitada em $\mathrm{Z}^{+} \mathrm{e}$ satisfaz $P w_{0}=0$. A norma do termo nào homogêneo é

$$
\begin{aligned}
& \mid \int_{0}^{1} D g\left(z_{n}(\xi)+\theta\left(z_{n}(\xi+h)-z_{n}(\xi)\right)\right) d \theta\left[z_{n}(\xi+h)-z_{n}(\xi)\right] \\
&\left.\quad-D g\left(z_{n}(\xi)\right) Z_{n}(\xi) h\right] \mid \\
&=\mid \int_{0}^{1} D g\left(z_{n}(\xi)+\theta\left(z_{n}(\xi+h)-z_{n}(\xi)\right)\right)-D g\left(z_{n}(\xi)\right) d \theta\left[z_{n}(\xi+h)-z_{n}(\xi)\right] \\
& \quad+D g\left(z_{n}(\xi)\right) w_{n} \mid \\
& \leq \quad \int_{0}^{1}\left|D g\left(z_{n}(\xi)+\theta\left(z_{n}(\xi+h)-z_{n}(\xi)\right)\right)-D g\left(z_{n}(\xi)\right)\right| d \theta\left|z_{n}(\xi+h)-z_{n}(\xi)\right| \\
& \quad+\left|D g\left(z_{n}(\xi)\right)\right|\left|w_{n}\right| \\
& \leq w(2 K|h|) 2 K|h|+\gamma\|w\|
\end{aligned}
$$

onde $\|w\|=\sup _{n \geq 0}|w|, w($.$) é o módulo de continuidade de D f(x)$ em $\left|x-x_{0}\right| \leq \Delta$ e usamos (4.8). Do Lema $1.1(i i)$, temos a estimativa

$$
\begin{aligned}
\|w\| & \leq K\left(1+e^{-\alpha}\right)\left(1-e^{-\alpha}\right)^{-1}[w(2 K|h|) 2 K|h| \mid+\gamma\|w\|] \\
& \leq 2 K^{2}\left(1+\epsilon^{-\alpha}\right)\left(1-e^{-\alpha}\right)^{-1} w(2 K|h|)|h|+\frac{1}{2}\|w\|
\end{aligned}
$$

Portanto,

$$
\|w\| \leq 4 K^{2}\left(1+e^{-\alpha}\right)\left(1-e^{-\alpha}\right)^{-1} w(2 K|h|)|h|
$$

Assim,

$$
\left|z_{n}(\xi+h)-z_{n}(\xi)-Z_{n}(\xi) h\right|=o(|h|) \text { quando }|h| \rightarrow 0,
$$

uniformemente em relação a $\xi$. Disto segue que para $n \geq 0, D z_{n}(\xi)=D Z_{n}(\xi)$ e, além disso, que $D z_{n}(\xi)$ é contínua em $\xi$. Também a condição de Lipschitz (4.8) implica que

$$
\left|D z_{n}(\xi)\right| \leq 2 K .
$$

Finalmente, quando $\xi=0, z_{n}(\xi)=0$ entâo a equaçào (4.9) toma a forma

$$
Z_{n+1}=D f\left(x_{0}\right) Z_{n} .
$$

Agora, $Z_{n}=D f^{n}\left(x_{0}\right) P$ é uma solução desta equação que é limitada em $\mathbf{Z}^{+} \mathrm{e}$ satisfaz $P Z_{0}=P$. Logo, pela unicidade,

$$
Z_{n}(0)=D f^{n}\left(x_{0}\right) P \quad(n \geq 0)
$$


Tomando $n=0$, temos

$$
Z_{0}(0)=D f^{0}\left(x_{0}\right) P=D I\left(x_{0}\right) P=P
$$

por outro lado,

$$
Z_{0}(0)=D z_{0}(0)=D v(0)
$$

portanto

$$
D v(0)=P
$$

Da definição de $v(\xi)$, temos

$$
v(\xi)=x_{0}+z_{n}(\xi) \Rightarrow D v(\xi)=D z_{n}(\xi)
$$

Logo $v(\xi)$ é $C^{1}$ (pois $z_{n}$ o é) e que os segundos ítens de (ii) e (iii) são satisfeitos.

Definição 4.1 Suponha que as hipóteses da Proposição 4.1 estejam satisfeitas. Então definimos a variedade estável local de $x_{0}$ como sendo o conjunto

$$
W_{l o c}^{s}\left(x_{0}\right)=\left\{v(\xi): \xi \in \mathcal{R}(P),|\xi|<\Delta / 4 K^{\prime}\right\}
$$

Definimos a variedade estável global de $x_{0}$ como sendo o conjunto

$$
W^{s}\left(x_{0}\right)=\left\{x \in \mathbf{R}^{p}: f^{n}(x) \rightarrow x_{0} \text { quando } n \rightarrow \infty\right\} .
$$

Lema 4.1 Suponha que as hipóteses da Proposição 4.1 estejam satisfeitas. Suponha que $x \in \mathbf{R}^{p}$ satisfaça

$$
\left|f^{n}(x)-x_{0}\right| \leq \Delta \text { para } n \geq 0
$$

Então temos a estimativa

$$
\left|f^{n}(x)-x_{0}\right| \leq\left(2 K+e^{\alpha}\right) e^{-\left[\alpha-\log \left(1+2 K \gamma e^{\alpha}\right)\right] n}\left|x-x_{0}\right| \text { para } n \geq 0
$$

onde

$$
\alpha-\log \left(1+2 K^{\prime} \gamma e^{\alpha}\right)>0
$$




\section{Prova:}

Primeiro, usando (4.1), temos

$1+2 K \gamma e^{\alpha}=1+e^{\alpha}\left(1-e^{-\alpha}\right)\left(1+e^{-\alpha}\right)^{-1}=1+\frac{e^{\alpha}-1}{1+e^{-\alpha}}=\frac{e^{-a}+e^{\alpha}}{1+e^{-\alpha}}<\frac{1+e^{\alpha}}{1+e^{-\alpha}}=e^{\alpha}$ o que implica em $\alpha-\log \left(1+2 K^{\prime} \gamma e^{\alpha}\right)>0$.

Agora tome $x$ satisfazendo (4.10). Então, $z_{n}=f^{n}(x)-x_{0}$ é uma solução da equação diferença (4.3) satisfazendo $\left|z_{n}\right| \leq \Delta$ para $n \geq 0$. Assim, a função de Green (veja Lema 1.1) para a equaçâo

$$
z_{n+1}=D f\left(x_{0}\right) z_{n}
$$

é dada por

$$
G(n, m)=\left\{\begin{array}{cc}
D f^{n-m}\left(x_{0}\right) P & \text { para } m \leq n \\
-D f^{n-m}\left(x_{0}\right)(I-P) & \text { para } n<m
\end{array}\right.
$$

$z_{n}$ é solução da equaçâo nâo homogênea

$$
z_{n+1}=D f\left(x_{0}\right) z_{n}+g\left(z_{n}\right)
$$

que é limitada em $n \geq 0$, e assim, segue de (1.13) na prova do Lema 1.1 que

$$
z_{n}=D f^{n}\left(x_{0}\right) P z_{0}+\sum_{m=0}^{n-1} D f^{n-m-1}\left(x_{0}\right) P g\left(z_{m}\right)-\sum_{m=n}^{\infty} D f^{n-m-1}\left(x_{0}\right)(I-P) g\left(z_{m}\right)
$$

para $n \geq 0$. Logo, para $n \geq 0$, temos a desigualdade

$$
\left|z_{n}\right| \leq K e^{-\alpha n}\left|z_{0}\right|+K \gamma\left\{\sum_{m=0}^{n-1} e^{-\alpha(n-m-1)}\left|z_{m}\right|+\sum_{m=n}^{\infty} e^{-\alpha(m+1-n)}\left|z_{m}\right|\right\}
$$

Para $n \geq 0$ tome

$$
\mu_{n}=\sup _{m \leq n}\left|z_{m}\right|
$$

Dado $n \geq 0$ e $\epsilon>0$, podemos encontrar $n^{\prime} \geq n$ tal que

$$
(1-\epsilon) \mu_{n} \leq\left|z_{n^{\prime}}\right|
$$

Então de (4.11), com $n^{\prime}$ no lugar de $n$,

$$
\begin{aligned}
(1-\epsilon) \mu_{n} & \leq K e^{-\alpha n^{\prime}}\left|z_{0}\right|+K \gamma\left\{\sum_{m=0}^{n^{\prime}-1} e^{-\alpha\left(n^{\prime}-m-1\right)}\left|z_{m}\right|+\sum_{m=n^{\prime}}^{\infty} e^{-\alpha\left(m+1-n^{\prime}\right)}\left|z_{m}\right|\right\} \\
& \leq K^{\prime} e^{-\alpha n}\left|z_{0}\right|+K \gamma\left\{\sum_{m=0}^{n-1} e^{-\alpha(n-m-1)}\left|z_{m}\right|\right.
\end{aligned}
$$




$$
\begin{aligned}
& \left.+\sum_{m=n} e^{-\alpha\left(n^{\prime}-m-1\right)}\left|z_{m}\right|+\sum_{m=n^{\prime}} e^{-\alpha\left(m+1-n^{\prime}\right)}\left|z_{m}\right|\right\} \\
\leq & K e^{-\alpha n}\left|z_{0}\right|+K \gamma\left\{\sum_{m=0}^{n-1} e^{-\alpha(n-m-1)}\left|z_{m}\right|\right. \\
& +K \gamma\left(1+e^{-\alpha}\right)\left(1-e^{-\alpha}\right)^{-1} \mu_{n} \\
\leq & K \epsilon^{-\alpha n}\left|z_{0}\right|+K \gamma \sum_{m=0}^{n-1} e^{-\alpha(n-m-1)}\left|z_{m}\right|+\frac{1}{2} \mu_{n}
\end{aligned}
$$

Portanto,

$$
\left(\frac{1}{2}-\epsilon\right) \mu_{n} \leq K e^{-\alpha n}\left|z_{0}\right|+K \gamma \sum_{m=0}^{n-1} e^{-\alpha(n-m-1)}\left|z_{m}\right|
$$

Isto ocorre para todo $\epsilon>0$. Faça $\epsilon \rightarrow 0$ para obter

$$
\left|z_{n}\right| \leq \mu_{n} \leq 2 K e^{-\alpha n}\left|z_{0}\right|+2 K \gamma \sum_{m=0}^{n-1} e^{-\alpha(n-m-1)}\left|z_{m}\right|
$$

para $n \geq 0$. Defina $w_{n}==e^{\alpha n}\left|z_{n}\right|$, entâo

$$
w_{n} \leq 2 K\left|z_{0}\right|+2 K \epsilon^{\alpha} \gamma \sum_{m+0}^{n-1} w_{m}
$$

para $n \geq 0$. Isto implica, por indução para $n \geq 1$, que

$$
w_{n} \leq\left(2 K\left|z_{0}\right|+2 K e^{a} \gamma w_{0}\right)\left(1+2 K e^{\alpha} \gamma\right)^{n-1}
$$

Portanto, para $n \geq 1$

$$
\begin{aligned}
\left|z_{n}\right| & \leq\left(2 K+2 K e^{\alpha} \gamma\right) e^{-\alpha n}\left(1+2 K e^{\alpha} \gamma\right)^{n-1}\left|z_{0}\right| \\
& =\frac{2 K+2 K e^{\alpha} \gamma}{1+2 K e^{\alpha} \gamma} e^{-\left[\alpha-\log \left(1+2 K e^{\alpha} \gamma\right)\right] n}\left|z_{0}\right| \\
& \leq\left(2 K+e^{\alpha}\right) e^{-\left[\alpha-\log \left(1+2 K e^{\alpha} \gamma\right)\right] n}\left|z_{0}\right|
\end{aligned}
$$


Proposição 4.2 Suponha que as hipóteses da Proposiçāo (4.1) estejam satisfeitas. Então

(i) $W_{l o c}\left(x_{0}\right)$ é uma subvariedade de $\mathbf{R}^{p}$;

(ii) $x \in W^{s}\left(x_{0}\right)$ se, e somente se, existe um inteiro $N$ tal que

$$
f^{N}(x) \in W_{l o c}^{s}\left(x_{0}\right)
$$

além disso, $f^{-N}\left(W_{l o c}^{s}\left(x_{0}\right)\right)$ é uma subvariedade de $\mathbf{R}^{p}$ contendo $x$ e contida em $W^{\prime}\left(x_{0}\right)$. Seu espaço tangente em $x$ é denotado por $T_{x} W^{\prime}\left(x_{0}\right)$ e é dado por

$$
T_{x} W^{s}\left(x_{0}\right)=\left\{\xi \in \mathbf{R}^{p}: \sup _{n \geq 0}\left|D f^{n}(x) \xi\right|<\infty\right\}
$$

\section{Prova:}

$W_{\text {loc }}^{s}\left(x_{0}\right)$ é imagem da aplicação $C^{\mathbf{1}} v(\xi)$ definida para $\xi$ em $\mathcal{R}(P)$, com $|\xi|<\Delta / 4 K$. Pela Proposição 4.1, v é 1-1. Também da prova da proposição $P D v(\xi)=P$ e assim $D v(\xi)$ é de posto máximo para todo $\xi$. Portanto, $W_{l o c}^{s}\left(x_{0}\right)$ é na verdade uma subvariedade de dimensão igual ao posto de $P$.

Segue da Proposição 4.1 que se $x \in W_{\text {loc }}^{s}\left(x_{0}\right)$ então para $n \geq 0$

$$
\left|f^{n}(x)-x_{0}\right| \leq \Delta
$$

Mas então segue do Lema 4.1 que $\left|f^{n}(x)-x_{0}\right| \rightarrow 0$ quando $n \rightarrow \infty$, logo que $x \in W^{s}\left(x_{0}\right)$. Portanto,

$$
W_{\text {loc }}^{s}\left(x_{0}\right) \subset W^{s}\left(x_{0}\right)
$$

Mais geralmente, se $f^{N}(x) \in W_{\text {loc }}^{s}\left(\dddot{x}_{0}\right)$ para algum $N$ então pelo que acabamos de provar $f^{N}(x) \in W^{s}\left(x_{0}\right)$ e assim $x \in W^{s}\left(x_{0}\right)$, pois de sua definiçào $W^{s}\left(x_{0}\right)$ é claramente um conjunto invariante.

Reciprocamente, suponha que $x \in W^{s}\left(x_{0}\right)$. Então existe $N$ tal que

$$
\left|f^{n}(x)-x_{0}\right|<\Delta / 4 K^{2} \text { para } n \geq N
$$

Isto implica que

$$
\left|f^{n}\left(f^{N^{\prime}}(x)\right)-x_{0}\right|<\Delta / 4 K^{2}<\Delta \text { para } n \geq 0
$$

Em particular, $\xi=P\left(f^{N}(x)-x_{0}\right)$ satisfaz $|\xi|<\Delta / 4 K$. Segue da Proposição $4.1(i)$ que $f^{N}(x)=v(\xi) \operatorname{com} \xi=P\left(f^{N}(x)-x_{0}\right)$. Logo $f^{N}(x) \in W_{\text {loc }}^{s}\left(x_{0}\right)$. 
Seja $x \in W^{s}\left(x_{0}\right)$. Então do que acabamos de provar existe um inteiro $N$ tal que $f^{N}(x) \in W_{i o c}^{i}\left(x_{0}\right)$. Assim $f^{-N}\left(W_{i o c}^{i}\left(x_{0}\right)\right)$ contém $x$ e é uma subvariedade de $\mathbf{R}^{p}$, pois $f^{-N}$ é um difeomorfismo e está contido em $W^{s}\left(x_{0}\right)$ pela primeira parte. Denotamos seu espaço tangente em $x$ por $T_{x} W^{s}\left(x_{0}\right)$ e entâo temos,

$$
T_{x} W^{s}\left(x_{0}\right)=D f^{-N}\left(f^{N}(x)\right)\left\{T_{f^{N}(x)} W_{l o c}^{s}\left(x_{0}\right)\right\}
$$

Agora, para qualquer $y$ em $W_{\text {loc }}\left(x_{0}\right), y=v(\xi)$ para algum $\xi \in \mathcal{R}(P)$, $|\xi|<\Delta / 4 K$ e

$$
T_{y} W_{\text {loc }}^{\text {: }}\left(x_{0}\right)=\mathcal{R}(D v(\xi))=\mathcal{R}\left(Z_{0}(\xi)\right)
$$

onde $Z_{n}(\xi)$ é a única solução de (4.9), a qual é limitada em $\mathbf{Z}^{+}$e satisfaz $P Z_{0}(\xi)=P$. Usando (4.4) e (4.7), vemos que (4.9) pode ser reescrito como

$$
Z_{n+1}=D f\left(f^{n}(y)\right) Z_{n}
$$

Como $f^{n}(y) \rightarrow x_{0}$ quando $n \rightarrow \infty$, segue como na prova da Proposição 3.1 que a equação variacional

$$
u_{n+1}=D f\left(f^{n}(y)\right) u_{n}
$$

tem uma dicotomia exponencial com projeções $P_{n}$ tendo o mesmo posto que $P$. Para qualquer vetor fixado $\eta, Z_{n}(\xi) \eta$ é uma solução de (4.13), limitada em $\mathbf{Z}^{+}$. Então pela Proposição 1.2

$$
Z_{0}(\xi) \eta \in \mathcal{R}\left(P_{0}\right)
$$

Isto ocorre para todo $\eta$, logo

$$
\mathcal{R}\left(Z_{0}(\xi)\right) \subset \mathcal{R}\left(p_{0}\right)
$$

Mas, como $P Z_{0}(\xi)=P, Z_{0}(\xi)$ tem o mesmo posto de $P$, e portanto, o mesmo posto de $P_{0}$. Logo,

$$
\mathcal{R}\left(Z_{0}(\xi)\right)=\mathcal{R}\left(P_{0}\right)
$$

Assim, usando a Proposição 1.2 e o fato que a matriz de transição para (4.13) é $D f^{n-m}\left(f^{m}(y)\right)$, temos

$$
T_{y} W_{\text {loc }}^{s}\left(x_{0}\right)=\mathcal{R}\left(P_{0}\right)=\left\{\xi \in \mathbf{R}^{p}: \sup _{n \geq 0}\left|D f^{n}(y) \xi\right|<\infty\right\}
$$

Então usando (4.12), temos

$$
T_{x} W^{s}\left(x_{0}\right)=\left\{D f^{-N}\left(f^{N}(x)\right) \xi: \xi \in \mathbf{R}^{p}, \sup _{n \geq 0}\left|D f^{n}\left(f^{N}(x)\right) \xi\right|<\infty\right\}
$$

Mas,

$$
D f^{n}\left(f^{N}(x)\right) \xi=D f^{n+N}(x) D f^{-N}\left(f^{N}(x)\right) \xi
$$


portanto,

$$
T_{z} W^{s}\left(x_{0}\right)=\left\{\xi \in \mathbf{R}^{p}: \sup _{n \geq 0}\left|D f^{n+N}(x) \xi\right|<\infty\right\}
$$

que é claramente igual a

$$
\left\{\xi \in \mathbf{R}^{p}: \sup _{n \geq 0}\left|D f^{n}(x)\right| \xi<\infty\right\}
$$

Observação 4.1 Seja $x_{0}$ um ponto fixo hiperbólico de um difeomorfismo $C^{1} f$ : $\mathbf{R}^{p} \rightarrow \mathbf{R}^{p}$. Definimos a variedade instável global de $x_{0}$ ao conjunto

$$
W^{u}\left(x_{0}\right)=\left\{x \in \mathbf{R}^{p}: f^{n}(x) \rightarrow x_{0} \text { quando } n \rightarrow-\infty\right\}
$$

Analogamente à Proposiçâo 4.1 podemos provar a existência de uma aplicação $C^{1}$ $w:\{\xi \in \mathcal{N}(P):|\xi|<\Delta / 4 K\} \rightarrow \mathbf{R}^{p}$ com a propriedade que $x=w(\xi)$ é o único $x$ tal que

$$
\begin{gathered}
(I-P)\left(x-x_{0}\right)=\xi \\
\left|f^{n}(x)-x_{0}\right| \leq \Delta \text { para } n \leq 0
\end{gathered}
$$

e também com propriedades análogas a (ii), (iii) e (iv). Entâo como na Proposição 4.2 podemos mostrar que $x \in W^{u}\left(x_{0}\right)$ se, e somente se, existe um inteiro $N$ tal que $f^{N}(x) \in W_{\text {loc }}^{u}\left(x_{0}\right)$, onde este último é a variedade local instável, isto é, a imagem da aplicação $w$. Além disso, se definirmos $T_{x} W^{u}\left(x_{0}\right)$ como o espaço tangente a $f^{-N}\left(W^{u}\left(x_{0}\right)\right)$ em $x$, entâo

$$
T_{x} W^{u}\left(x_{0}\right)=\left\{\xi \in \mathbf{R}^{p}: \sup _{n \leq 0}\left|D f^{n}(x) \xi\right|<\infty\right\}
$$

Teorema 4.1 Seja $x_{0}$ um ponto fixo hiperbólico de um difeomorfismo $C^{1}$ $f: \mathbf{R}^{p} \rightarrow \mathbf{R}^{p}$ e sejam $W^{s}\left(x_{0}\right), W^{u}\left(x_{0}\right)$ as variedades estável e instável global de $x_{0}$. Entâo yo $\dot{\epsilon}$ um ponto homoclínico com respeito a $x_{0}$ se, e somente se $y_{0} \neq x_{0} e$

$$
y_{0} \in W^{s}\left(x_{0}\right) \cap W^{u}\left(x_{0}\right)
$$

$y_{0}$ é transversal (de acordo com a Definição 3.3) se, $\epsilon$ somente se,

$$
T_{y_{0}} W^{s}\left(x_{0}\right) \cap T_{y_{0}} W^{u}\left(x_{0}\right)=\{0\}
$$




\section{Prova:}

0 fato que $y_{0}$ é um ponto homoclínico (de acordo com a Definição 3.2) se, e somente se, $y_{0} \neq x_{0}$ e $y_{0} \in W^{s}\left(x_{0}\right) \cap W^{u}\left(x_{0}\right)$ segue diretamente da definição . Agora, a solução $u_{n}$ da equação variacional (3.3) satisfazendo $u_{0}=\xi$ é dada por $u_{n}=D f^{n}\left(y_{0}\right) \xi$. Claramente entào $u_{n}$ é limitada em Z se, e somente se,

$$
\xi \in T_{y_{0}} W^{s}\left(x_{0}\right) \cap T_{y_{0}} W^{u}\left(x_{0}\right)
$$

Assim, (3.3) não tem uma solução nâo trivial limitada em $\mathbf{Z}$, isto é, $y_{0}$ é uma ponto homoclínico transversal de acordo com a Definição 3.3 se, e somente se,

$$
T_{y_{0}} W^{s}\left(x_{0}\right) \cap T_{y_{0}} W^{u}\left(x_{0}\right)=\{0\} .
$$

Teorema 4.2 Próximo a qualquer ponto homoclínico transversal existem infinitos outros pontos homoclínicos transversais.

\section{De fato,}

Seja $y_{0}$ um ponto homoclinico transversal com respeito a um ponto fixo hiperbólico $x_{0}$.

Seja $\Delta>0$ como Proposição 4.1. Na prova do Teorema 3.1 tome $r=m-1$ e $p_{1}=0$. Escolha $N_{1}$ tal que

$$
\left|f^{n}\left(y_{0}\right)-x_{0}\right| \leq \frac{\left|x_{0}-y_{0}\right|}{2} \text { se }|n|>N_{1}
$$

Também escolha $\epsilon>0$ tal que ele satisfaça às condições adicionais

$$
\begin{gathered}
\epsilon \leq \Delta \\
\epsilon \leq \frac{\left|x_{0}-y_{0}\right|}{4} \\
\epsilon \leq \frac{1}{2} \min \left\{\left|f^{i}\left(y_{0}\right)-y_{0}\right|: 0<|i| \leq N_{1}\right\}
\end{gathered}
$$

Seja $q$ um inteiro positivo e considere a bisequência em $\mathcal{I}_{m}$

$$
a=(\ldots, m, m, 1, \ldots, 1, m, m, \ldots)
$$


onde existem $q$ l's e $a_{0}$ é o primeiro 1. Pelo shadowing lemma existe uma única órbita que $\epsilon$-shadows a pseudo-órbita formada pelos segmentos

$$
\ldots, C_{m}, C_{a_{0}}=C_{1}, \ldots, C_{1}, C_{m}, \ldots
$$

Seja $x^{(q)}$ o ponto na órbita que 'sombreia' o ponto médio de $y_{0}$ de

$$
C_{a_{0}}=C_{1}=\left\{f^{-N}\left(y_{0}\right), \ldots, y_{0}, \ldots, f^{N}\left(y_{0}\right)\right\}
$$

Então

$$
\left|x^{(q)}-y_{0}\right|<\epsilon
$$

Como

$$
C_{m}=\left\{x_{0}, \ldots, x_{0}, \ldots, x_{0}\right\}
$$

para $|n|$ suficientemente grande

$$
\left|f^{n}\left(x^{(q)}\right)-x_{0}\right|<\epsilon \leq \Delta
$$

Logo, pelo Lema $4.1, f^{n}\left(x^{(q)}\right) \in W^{s}\left(x_{0}\right)$ para $n>0$ suficientemente grande. Como $W^{s}\left(x_{0}\right)$ é invariante, isto significa que $x^{(q)} \in W^{s}\left(x_{0}\right)$. Analogamente provamos que $x^{(q)} \in W^{u}\left(x_{0}\right)$. Portanto,

$$
x^{(q)} \in W^{s}\left(x_{0}\right) \cap W^{u}\left(x_{0}\right)
$$

Também como $\left|x^{(q)}-y_{0}\right|<\epsilon e\left|x_{0}-y_{0}\right| \geq 4 \epsilon, x^{(q)} \neq x_{0}$. Logo $x^{(q)}$ é um ponto homoclínico .

Que a equação variacional

$$
u_{n+1}+D f^{n}\left(x^{(q)}\right) u_{n}
$$

tem uma dicotomia exponencial segue da Observação 2.2 ( $\epsilon$ e $\delta$ podem ser tomados menores para assegurar isto). Então $x^{(q)}$ é um ponto homoclínico transversal.

Agora, cada ponto na órbita de $x^{(q)} \epsilon$-shadows $x_{0}$ ou um dos pontos $f^{i}\left(y_{0}\right)$, $|i| \leq N$. Nossas hipóteses sobre $\epsilon$ e $N_{1}$ implicam que se um ponto $\epsilon$-shadows $x_{0}$ ou $f^{i}\left(y_{0}\right)$ onde $0<|i| \leq N$, então ele não pode $\epsilon$-shadows $y_{0}$. Na verdade, existem exatamente $q$ pontos na órbita de $x^{(q)}$ que $\epsilon$-shadows $y_{0}$. Portanto, se $q_{1} \neq q_{2}$ as órbitas de $x^{\left(q_{1}\right)}$ e $x^{\left(q_{2}\right)}$ nâo se intersectam.

Portanto, mostramos que a bola de rajo $\epsilon$ centrada em $y_{0}$ contém uma quantia enumerável de pontos homoclinicos transiversais, nenhum deles está na órbita de qualquer outro. 
Teorema 4.3 Se o difeomorfismo $C^{1} f: \mathbf{R}^{p} \rightarrow \mathbf{R}^{p}$ tem um ponto homoclínico transversal e $g: \mathbf{R}^{p} \rightarrow \mathbf{R}^{p}$ é outro difeomorfismo que está $C^{1}$-próximo a $f$, então g também tem um ponto homoclínico transversal.

De fato, Seja $x_{0}$ um ponto fixo hiperbólico para $f$. Então existem constantes $K, \alpha$ e um projeção $P$ tal que as desigualdades (3.1) ocorrem. Suponha também que $y_{0}$ seja um ponto homoclínico transversal com respeito a $x_{0}$, ou seja, $f^{n}\left(y_{0}\right) \rightarrow x_{0}$ quando $|n| \rightarrow \infty$ e a equação variacional (3.3) tem um dicotomia exponencial em $\mathrm{Z}$ com constantes que podemos assumir serem $K, \alpha$ também.

Seja $g: \mathbf{R}^{p} \rightarrow \mathbf{R}^{p}$ um difeomorfismo $C^{1}$ tal que

$$
\begin{gathered}
|g(x)-f(x)| \leq \epsilon \text { para } x \in S \\
|D g(x)-D f(x)| \leq \epsilon \text { para }|x-y| \leq \Delta_{0}, y \in S
\end{gathered}
$$

onde $S=\left\{x_{0}\right\} \cup\left\{f^{n}\left(y_{0}\right): n \in \mathbf{Z}\right\}, \Delta_{0}$ é uma constante positiva e as condições serão determinadas abaixo.

Primeiro mostramos que quando $\epsilon$ é suficientemente pequeno, $g$ tem um ponto fixo hiperbólico próximo a $x_{0}$. Fazemos isto mostrando que a equação diferença

$$
x_{n+1}=g\left(x_{n}\right)
$$

tem uma única solução $x_{n}$ próxima a $x_{0}$. Se colocarmos

$$
x_{n}=x_{0}+z_{n},
$$

então $z_{n}$ será uma solução da equação

$$
z_{n+1}=D f\left(x_{0}\right) z_{n}+G\left(z_{n}\right)
$$

onde

$$
G(z)=f\left(x_{0}+z\right)-f\left(x_{0}\right)-D f\left(x_{0}\right) z+g\left(x_{0}+z\right)-f\left(x_{0}+z\right)
$$

Vemos que

$$
|G(0)|=\left|g\left(x_{0}\right)-f\left(x_{0}\right)\right| \leq \epsilon
$$

e que quando $|z| \leq \Delta_{0}$

$$
\begin{aligned}
|D G(z)| & \leq\left|D f\left(x_{0}+z\right)-D f\left(x_{0}\right)\right|+\left|D g\left(x_{0}+z\right)-D f\left(x_{0}+z\right)\right| \\
& \leq w(|z|)+\epsilon
\end{aligned}
$$

onde $w($.$) é o módulo de continuidade de D f(x)$ no conjunto $\left\{x \in \mathbf{R}^{p}:|x-y| \leq \Delta_{0}\right.$ para algum $\left.y \in S\right\}$. 
Assumamos que

$$
\Delta(\epsilon)=2 K\left(1+e^{-\alpha}\right)\left(1-\epsilon^{-\alpha}\right)^{-1} \epsilon \leq \Delta_{0}
$$

Então se

$$
2 K\left(1+e^{-\alpha}\right)\left(1-e^{-\alpha}\right)^{-1}(w(\Delta(\epsilon))+\epsilon) \leq 1
$$

segue da Proposição 1.4 que a equação (4.17) tem uma única solução $z_{n}$ tal que

$$
\left|z_{n}\right| \leq \Delta(\epsilon)
$$

para todo $n$. Isto significa que $x_{n}=x_{0}+z_{n}$ é a única solução da equação (4.16) tal que

$$
\left|x_{n}-x_{0}\right| \leq \Delta(\epsilon)
$$

para todo $n$. Mas $x_{n+1}$ claramente é também uma solução . Pela unicidade devemos ter $x_{n+1}=x_{n}$ para todo $n$. Assim, $x_{n}$ é, na verdade, uma constante $x_{0}{ }^{\prime}$. $x_{0}$ ' é então um ponto fixo de $g$ satisfazendo

$$
\left|x_{0}^{\prime}-x_{0}\right| \leq \Delta(\epsilon)
$$

Além disso,

$$
\begin{aligned}
\left|D g\left(x_{0}^{\prime}\right)-D f\left(x_{0}\right)\right| & \leq D g\left(x_{0}^{\prime}\right)-D f\left(x_{0}^{\prime}\right)|+| D f\left(x_{0}^{\prime}\right)-D f\left(x_{0}\right) \mid \\
& \leq \epsilon+w(\Delta(\epsilon))
\end{aligned}
$$

Então segue da Proposição 1.5 com $\delta=\alpha / 2$ que se (4.19) é satisfeita e também

$$
2 K e^{\alpha}\left(e^{-\frac{o}{2}}+1\right)\left(e^{\frac{o}{2}}-1\right)^{-1}[\epsilon+w(\Delta(\epsilon)] \leq 1
$$

a equação linear

$$
u_{n+1}=D g\left(x_{0}^{\prime}\right) u_{n}
$$

tem uma dicotomia exponencial em $\mathbf{Z}$ com constantes $K_{1}$ e $\alpha / 2$ onde $K_{1}=$ $2 K\left(1+e^{-\frac{a}{2}}\right)\left(1-e^{-\frac{a}{2}}\right)^{-1}$. Então a Observação 1 nos diz que os autovalores de $D g\left(x_{0}^{\prime}\right)$ estão fora do círculo unitário e que se $P^{\prime}$ é a projeçâo com imagem a soma dos autoespaços generalizados correpondentes aos autovalores dentro do círculo unitário e com núcleo a soma dos autoespaços generalizados correspondentes aos autovalores fora do círculo unitário, as desigualdades

$$
\begin{gathered}
\left|D g^{n}\left(x_{0}^{\prime}\right) P^{\prime}\right| \leq K_{1}^{\prime} e^{-\frac{\alpha}{2} n} \quad(n \geq 0) \\
\left|D g^{n}\left(x_{0}^{\prime}\right)\left(I-P^{\prime}\right)\right| \leq K_{1} e^{\frac{a}{2} n} \quad(n \leq 0)
\end{gathered}
$$

são satisfeitas. Logo $x_{0}^{\prime}$ é certamente um ponto fixo hiperbólico de $g$. 
Mostraremos agora que para $\epsilon$ suficientemente pequeno, $g$ tem um ponto homoclínico transversal $y_{0}^{\prime}$ próximo a $y_{0}$. Faremos isto encontrando uma solução $x_{n}$ da equação (4.16) próxima a $f^{n}\left(y_{0}\right)$. Se escrevermos

$$
x_{n}=f^{n}\left(y_{0}\right)+z_{n}
$$

então $z_{n}$ deve ser uma soluçâo da equação

$$
z_{n+1}=D f\left(f^{n}\left(y_{0}\right)\right) z_{n}+G_{n}\left(z_{n}\right)
$$

onde

$G_{n}(z)=f\left(f^{n}\left(y_{0}\right)+z\right)-f\left(f^{n}\left(y_{0}\right)\right)-D f\left(f^{n}\left(y_{0}\right)\right) z+g\left(f^{n}\left(y_{0}\right)+z\right)-f\left(f^{n}\left(y_{0}\right)+z\right)$

Note que

$$
\left|G_{n}(0)\right|=\left|g\left(f^{n}\left(y_{0}\right)\right)-f\left(f^{n}\left(y_{0}\right)\right)\right| \leq \epsilon
$$

e se $|z| \leq \Delta_{0}$

$$
\begin{aligned}
\left|D G_{n}(z)\right| \leq & \left|D f\left(f^{n}\left(y_{0}\right)+z\right)-D f\left(f^{n}\left(y_{0}\right)\right)\right| \\
& +\left|D g\left(f^{n}\left(y_{0}\right)+z\right)-D f\left(f^{n}\left(y_{0}+z\right)\right)\right| \\
\leq & w(|z|)+\epsilon
\end{aligned}
$$

Então segue da Proposição 1.4 que se as condições (4.18), (4.19) estão satisfeitas, a equação (4.21) tem uma única solução $z_{n}$ tal que

$$
\left|z_{n}\right| \leq \Delta(\epsilon)
$$

para todo $n$.

Escolha $\Delta_{1}>0$ tal que

$$
K_{1}=4 K_{1}\left(1+e^{-\frac{a}{2}}\right)\left(1-e^{-\frac{a}{2}}\right)^{-1} w\left(\Delta_{1}\right) \leq 1
$$

e assuma que

$$
\begin{gathered}
8 K_{1}\left(1+\epsilon^{-\frac{a}{2}}\right)\left(1-e^{-\frac{a}{2}}\right)^{-1} \epsilon \leq 1 \\
3 \Delta(\epsilon) \leq \Delta_{1}
\end{gathered}
$$

Então se $\left|x-x_{0}^{\prime}\right| \leq \Delta_{1}$,

$$
\begin{aligned}
\left|D g(x)-D g\left(x_{0}^{\prime}\right)\right| \leq & |D g(x)-D f(x)|+\left|D f(x)-D f\left(x_{0}^{\prime}\right)\right| \\
& +\left|D f\left(x_{0}^{\prime}\right)-D g\left(x_{0}^{\prime}\right)\right| \\
\leq & \epsilon+w\left(\Delta_{1}\right)+\epsilon \\
\leq & \gamma_{1}=\frac{1}{2} K_{1}^{-1}\left(1+e^{-\frac{a}{2}}\right)^{-1}\left(1-e^{-\frac{a}{2}}\right)
\end{aligned}
$$


Isto significa que podemos aplicar a Proposição 4.1 e o Lema 4.1 a $g$ e $x_{0}^{\prime}$ com os valores $K_{1}, \alpha / 2, \Delta_{1}, \gamma_{1}$ no lugar de $K, \alpha, \Delta, \gamma$.

Existe um inteiro positivo $N$ tal que

$$
\left|f^{n}\left(y_{0}\right)-x_{0}\right| \leq \frac{\Delta_{1}}{3}
$$

para $|n| \geq N$. Então se $|n| \geq N$,

$$
\begin{aligned}
\left|g^{n}\left(y_{0}^{\prime}\right)-x_{0}^{\prime}\right| & \leq\left|g^{n}\left(y_{0}^{\prime}\right)-f^{n}\left(y_{0}\right)\right|+\left|f^{n}\left(y_{0}\right)-x_{0}\right|+\left|x_{0}^{\prime}-x_{0}\right| \\
& \leq \Delta(\epsilon)+\frac{\delta_{1}}{3}+\Delta(\epsilon) \leq \Delta_{1}
\end{aligned}
$$

Segue então do Lema 4.1 que $g^{N}\left(y_{0}^{\prime}\right) \in W^{s}\left(x_{0}^{\prime}\right)$ e portanto que $y_{0}^{\prime} \in W^{s}\left(x_{0}^{\prime}\right)$. Analogamente, $y_{0}^{\prime} \in W^{u}\left(x_{0}^{\prime}\right)$. Se assumirmos

$$
2 \Delta(\epsilon)<\left|y_{0}-x_{0}\right|
$$

então

$$
\left|y_{0}^{\prime}-x_{0}^{\prime}\right| \geq\left|y_{0}-x_{0}\right|-\left|y_{0}^{\prime}-y_{0}\right|-\left|x_{0}^{\prime}-x_{0}\right| \geq\left|y_{0}-x_{0}\right|-2 \Delta(\epsilon)>0 .
$$

Assim, $y_{0}^{\prime} \neq x_{0}^{\prime}$ e $y_{0}^{\prime}$ é portanto um ponto homoclínico com respeito à $x_{0}^{\prime}$.

Além disso, para todo $n$

$$
\begin{aligned}
\left|D g\left(g^{n}\left(y_{0}^{\prime}\right)\right)-D f\left(f^{n}\left(y_{0}\right)\right)\right| \leq & \left|D g\left(g^{n}\left(y_{0}^{\prime}\right)\right)-D f\left(g^{n}\left(y_{0}^{\prime}\right)\right)\right| \\
& +\left|D f\left(g^{n}\left(y_{1}^{\prime}\right)\right)-D f\left(f^{n}\left(y_{0}\right)\right)\right| \\
\leq & \epsilon+w(\Delta(\epsilon))
\end{aligned}
$$

Como (3.3) tem uma dicotomia exponencial em $\mathbf{Z}$ com constantes $K, \alpha$ e (4.19), (4.20) são satisfeitas, segue da Proposição 1.5 que a equação variacional

$$
u_{n+1}=D g\left(g^{n}\left(y_{0}^{\prime}\right)\right) u_{n}
$$

tem uma dicotomia exponencial em Z. Portanto, $y_{0}^{\prime}$ é um ponto homoclínico transversal.

Concluindo, mostramos que se $g: \mathbf{R}^{p} \rightarrow \mathbf{R}^{p}$ é um difeomorfismo satisfazendo (4.14), (4.15), onde $\epsilon$ satisfaz (4.18), (4.19), (4.20), (4.22), (4.23) e (4.24), então $g$ tem um ponto homoclínico transversal. 


\section{Bibliografia}

[1] D. V. Anosov, Geodesic Flows on Compact Riemannian Manifolds of Negative Curvature. Proc. Steklov Inst. Math., 90 (1967); English transl., Amer. Math. Soc. Transl. (1969).

[2] J. Guckenheimer, J. Moser e S. Newhouse, Dynamical Systems, Birkhauser, Boston (1980).

[3] J. MOSER, Stable and Random Motions in Dynamical Systems. Princeton Univ. Press, Princeton, NJ (1973).

[4] Z. Nitecki, Differentiable Dynamics - An Introduction to the Orbit Struture of Diffeomorphisms. The M.I.T. Press (1971).

[5] K. J. Palmer, Exponential Dichotomies, the Shadowing Lemma and Transversal Homoclinic Points. Dynamics Reported. Volume 1, (1988), 265306.

[6] K. J. Palmer, Exponential Dichotomy and Transversal Homoclinic Points. J. Differential Equations, 55, (1984), 225-256.

[7] C. Robinson, Stability Theorems and Hyperbolicity in Dynamical Systems. Rocky Mountains J. Math., 35, (1983), 98-103.

[8] S. Smale, Diffeomorphisms with Many Periodic Points. Differential and Combinatorial Topology, S. Cairns (ed.), Princeton Univ. Press, Princeton, $\mathrm{NJ},(1965), 63-80$.

[9] S. Smale, Differentiable Dynamical Systems. Bull. Amer. Math. Soc., 73, (1967), 747-817. 